Old Dominion University

ODU Digital Commons

2020

Boundary Vortex Formation in Polarization-Modulated Orthogonal Smectic Liquid Crystals

Carlos J. García-Cervera

Tiziana Giorgi

Sookyung Joo

Follow this and additional works at: https://digitalcommons.odu.edu/mathstat_fac_pubs

Part of the Applied Mathematics Commons 


\title{
BOUNDARY VORTEX FORMATION IN POLARIZATION-MODULATED ORTHOGONAL SMECTIC LIQUID CRYSTALS
}

\author{
CARLOS J. GARCíA-CERVERA*, TIZIANA GIORGI ${ }^{\dagger}$, AND SOOKYUNG JOO ${ }^{\ddagger}$
}

\begin{abstract}
We investigate the relaxation of an energy functional originated in the physics literature to study the bistability of polarization modulated orthogonal smectic phases (SmAP Fmod) of bent-core molecules liquid crystals. We show that the interplay between the mixed boundary conditions and the shape of the sample results in boundary defects. We also analyze the bistable switching due to an applied electric field via gradient flow numerical simulations. Our computations reveal a novel dynamic scenario, where switching is achieved by the formation of two internal vortices.
\end{abstract}

1. Introduction. A liquid crystal (LC) is a material that exhibits states between liquid and crystal, where it loses some or all of its positional order, but maintains partial orientational order. In the nematic phase, only some orientational order is preserved, and molecules tend to locally orient along a preferred direction, represented by a unit vector, $n$, called director. As temperature decreases the mechanical interactions become more pronounced, some degree of positional order appears, and layered structures form, entering the realm of smectic phases. Two common smectic phases are the smectic $\mathrm{A}(\mathrm{SmA})$, and the smectic $\mathrm{C}(\mathrm{SmC})$, with their chiral variants, $\mathrm{SmA}^{*}$ and $\mathrm{SmC}^{*}$. In the $\mathrm{SmA}$ phase, the axes of the orientational and positional orders are parallel, while in the $\mathrm{SmC}$ they are tilted one with respect to the other.

At the base of most practical uses of LC is the observation that the local orientation can be changed by applying an electric or a magnetic field. At the moment, display devices are mostly based on nematic LCs, but operate close to physical limits, [33]. Hence, the interest in smectics, which have potential for superior operational speed and resolution, [19]. An attractive feature of the SmA phase is its high contrast ratio, and excellent bistability [22, 21, 27]. Bistability means that there are two energetically equivalent global minimal states, which implies the possibility of maintaining a static image without requiring a continuous source of power $[12,6,7]$. Thus, bistable LCs capable of SmA-like phases are desirable for devices, such as mobile phones and large area signages, where saving power consumption is critical. The available bistable devices are based on nematic chiral or ferroelectric $\mathrm{SmC}^{*} \mathrm{LCs}$ [8]. But, synthesizing chiral molecules is expensive, and for the past decade [25, 9, 17] scientists have been extensively studying LCs composed of achiral bow-like shaped molecules, so-called bent-core liquid crystals (BLCs), where the bent shape allows for efficient packing, and results in ferroelectric properties. And, while ferroelectricity in rod-like LCs is caused by the tilt of the molecules with respect to the smectic layer normal, thus it is present only in $\mathrm{SmC}^{*}$ phases, the layers of $\mathrm{BLC}$ compounds can be

*Mathematics Department, University of California, Santa Barbara, CA 93106 (cgarcia@math.ucsb.edu) and Visiting Professor at BCAM - Basque Center for Applied Mathematics, Mazarredo 14, E48009 Bilbao, Basque Country, Spain. This material is based upon work supported by Bizkaia Talent and European Commission through COFUND Programme, under Award No. AYD-000-284 and with the collaboration of BCAM.

${ }^{\dagger}$ Department of Mathematical Sciences, New Mexico State University, Las Cruces, NM 88001 (tgiorgi@nmsu.edu). Funding to this author was provided by the National Science Foundation Grant \#DMS-1909273

${ }^{\ddagger}$ Department of Mathematics and Statistics, Old Dominion University, Norfolk, VA 23529 (sjoo@odu.edu). Funding to this author was provided by the National Science Foundation Grant \#DMS-1909268 and Simons Foundation Grant No. 422622 
polar also in the SmA-like phase (SmAP). The discovery of a ferroelectric SmA phase $\left(\mathrm{SmAP}_{\mathrm{F}}\right)$ in BLCs, announced in a 2011 Science Magazine article [28], see also [16], introduced scientists to the first experimentally confirmed "proper fluid ferroelectric", [26]. A one-dimensional continuum model for this phase is presented in [14].

We are interested in the ferroelectric bistability of polarization-modulated orthogonal smectic LCs described first in [36], where the authors report a BLC compound exhibiting a $\mathrm{SmAP}_{\mathrm{F}}$ phase at a lower temperature, and a polarization splay-modulated orthogonal $\mathrm{SmA}$ phase $\left(\mathrm{SmAP}_{\mathrm{Fmod}}\right)$ at a higher temperature. The tendency to splay results in a periodically splay-modulated bulk phase, where each splay domain is separated by a domain wall, and which experiments show has a bistable response to an applied electric field. In [36] is theorized that opposite anchoring at the stripe boundaries, and in-polarization form topological singularities (Figure 1) leading to bistability, and a one-dimensional energy is introduced to model this effect.

We study a two-dimensional version of a relaxation of the energy proposed in [36], see also the dimensional reduction argument presented in [11], which depends on a physical parameter, $\varepsilon$, and is defined on a square domain, $\Omega$, modeling the cross section of one thin splay domain. Adapting, and generalizing methods from [32], [1] and [2], we prove that for small values of $\varepsilon$, global energy minimizers, $p^{\varepsilon}=\left(p_{1}^{\varepsilon}, p_{2}^{\varepsilon}\right)$, of this energy (denoted by $E_{\varepsilon}$ ) converge up to subsequences to an $S^{1}$ valued function, which has always boundary vortices, but never internal ones. Converging subsequences exhibit at $\varepsilon$-distance from the boundary, and having location consistent with the formation of boundary vortices located on opposite sides of the sample away from the vertices of the domain, what we informally call near defects. These are $\varepsilon$-dependent sets where the relaxed polarization director has modulus less than one-half, roughly speaking, their union is the set $S_{\varepsilon}$ defined in (4.1), and which we expect will contain the vortices of the limiting problem. Our main analytical result reads as follows:

Theorem 4.3: Let $p^{\varepsilon}$ be a minimizer of $E_{\varepsilon}$. For any sequence $\varepsilon \rightarrow 0$, there is a subsequence $\varepsilon_{n} \rightarrow 0$, and two points $\left\{b_{1}^{*}, b_{2}^{*}\right\}$, on opposite sides of the horizontal boundary, $\Gamma_{H}$, such that $p^{\varepsilon_{n}} \rightarrow p^{*}$ in $H_{l o c}^{1} \cap C_{l o c}\left(\bar{\Omega} \backslash\left\{b_{1}^{*}, b_{2}^{*}\right\}\right)$, and $p^{*}: \bar{\Omega} \backslash\left\{b_{1}^{*}, b_{2}^{*}\right\} \rightarrow \mathbb{S}^{1}$ satisfies the equation $\Delta p^{*}+\left|\nabla p^{*}\right|^{2} p^{*}=\sigma\left(\sigma_{s} p_{2}^{*} p^{*}-\mathbf{e}\right)$. Furthermore, $p^{*}$ is a piecewise constant vector field on $\bar{\Gamma}_{H} \backslash\left\{b_{1}^{*}, b_{2}^{*}\right\}$, with values $\pm \mathbf{e}_{1}$; and $p^{*}=\nu$ on the vertical boundary, $\Gamma_{V}$. Additionally, $\sigma_{s} p_{2}^{*} \geq 0$ a.e. for $\sigma \neq 0$. In here, $\sigma>0$ is proportional to the applied field, $\sigma_{s}$ is the sign of the applied field, and $\mathbf{e}=\sigma_{s} \mathbf{e}_{2}$.

Previous studies on boundary vortices formation in Ginzburg-Landau-type models $[24,18,1,2]$ deal with smooth domains, and the presence of defects is a result of the imposed boundary conditions, and values of physical parameters. Instead, in here, the interplay of boundary conditions and shape of the domain is essential for the formation and location of the boundary vortices, and for the absence of global minimizers which have internal vortices. And, novel ideas are needed to estimate the energy signature of defects at and near the corners (see Propositions 3.5 and 3.6), to deal with different boundary conditions (Dirichlet and Robin) in different parts of the boundary.

We also numerically study the dynamics of the model bistability, that is we examine how the polarization is switched when the direction of the applied electric field is reversed. While one might expect that the switching happens with the nucleation of one internal vortex, which then travels from one side to the other, [36]; our simulations suggest the formation of two distinct internal vortices, which move towards the center of the sample, where they annihilate with each other, see Figures 5 and 6 .

The paper is organized as follows. In Section 2, we introduced the mathematical setup needed to study the proposed energy functional. Section 3 contains the deriva- 
tion of an energy upper bound, and standard estimates used to obtain the convergence result of Theorem 4.3. In Section 4, we derive a matching energy lower bound, and show that up to subsequences global minimizers have only near boundary defects. Finally, in Section 5 we present our gradient flow numerical simulations.

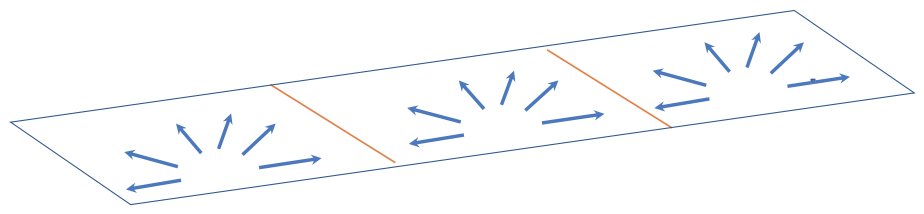

FIG. 1. Polarization splay stripes

2. Model. The bow-like shape of BLCs is described by the nematic director $n$, directed along the molecular axis, and the polarization director $p$, along the bow of the molecules; which is the same as the direction of the spontaneous polarization, $\mathbf{P}=P p$, and is perpendicular to $n$. Hence, the constraint $n \cdot p=0$. The layers of smectic phases are model through the so-called smectic order parameter $\Psi$.

To describe the experiments in [36], we start from an energy in terms f $\Psi, n$ and $p$; and, as in [36], we assume $\mathbf{P}=P_{0} p$, with $P_{0}$ constant; $n=\mathbf{e}_{3}$; and $\Psi$ constant. Since $n$ and $p$ are constrained by $n \cdot p=0$, these assumptions imply that only in-layer polarization is possible, i.e. $p \in \mathbb{S}^{1}$, and $p=\left(p_{1}, p_{2}, 0\right)$. Again following [36], we also assume constant applied electric field. In conclusion, we have: $\mathbf{E}_{e x}=E_{e x} \mathbf{e}_{2}, n=$ $\mathbf{e}_{3}, \mathbf{P}=P_{0} p$ with $|p|=1$, and $E_{e x}$ and $P_{0}$ fixed constants. Note that the applied electric field tends to make the polarization orient along the $y$ direction.

The intrinsic property of the $\mathrm{SmAP}_{\mathrm{Fmod}}$ phase, where a periodic array of domains with linear polarization splay is formed, gives rise to opposite strong anchoring, see Figure 1. In other words, the polarization splay stripes are separated by domain walls, where the polarization has opposite direction on each side of the wall. This suggests that one polarization splay stripe can be modeled by a square domain with polar director pointing in opposite directions on the vertical sides of the sample.

Following [36] and [11], we adopt the free energy:

$$
E(p)=\int_{\Omega_{S}}\left(K|\nabla p|^{2}-P_{0} E_{e x} p_{2}\right) d \mathbf{x}+W \int_{\Gamma_{S H}}(p \cdot \nu)^{2},
$$

where $\Omega_{S}=(0, S)^{2}$ and $\Gamma_{S H}=(0, S) \times\{0, S\}$. We then rescale length by $S$, to rewrite the energy in dimensionless units:

$$
\frac{E(p)}{K}=\int_{\Omega}\left(|\nabla p|^{2}-2 \sigma \sigma_{s} p_{2}\right) d \mathbf{x}+\frac{1}{\varepsilon} \int_{\Gamma_{H}}(p \cdot \nu)^{2},
$$

with $\Omega=(0,1)^{2}, \Gamma_{H}=(0,1) \times\{0,1\}, \sigma=\frac{S^{2} P_{0}\left|E_{e x}\right|}{2 K}, \sigma_{s}=\operatorname{sign}\left(E_{e x}\right), \varepsilon=\frac{K}{W S}$.

We assume Dirichlet boundary condition for $p$ on the vertical sides:

$$
p=\nu \quad \text { for } x \in \Gamma_{V}=\{0,1\} \times(0,1),
$$

where $\nu$ is the outward normal vector. The surface energy on the horizontal part of the boundary is included to model the polarization structure along the stripe boundaries. Part of this term models the electric self-interaction energy presented in [3], see [11]. 
The explanation proposed in [36], for the mechanism driving the bistable response to an applied electric field of the $S m A P_{F m o d}$ phase, envisions vortices moving from one side of the horizontal part of the boundary to the other. However, it is known that an interior vortex for a map $p \in \mathbb{S}^{1}$ has infinite energy [5]. We follow the standard literature in the Ginzburg-Landau theory, and relax the $p \in \mathbb{S}^{1}$ constraint. Up to constants, we arrive to the following energy functional:

$$
E_{\varepsilon}(p)=\frac{1}{2} \int_{\Omega}\left(|\nabla p|^{2}+\frac{1}{2 \varepsilon^{2}}\left(1-|p|^{2}\right)^{2}+\sigma|\mathbf{e}-p|^{2}\right) d x d y+\frac{1}{2 \varepsilon} \int_{\Gamma_{H}}(p \cdot \nu)^{2} d x
$$

where $\mathbf{e}=\sigma_{s} \mathbf{e}_{2}$. We impose the Dirichlet boundary condition (2.1), and define

$$
H_{D}^{1}=\left\{p \in H^{1}\left(\Omega ; \mathbb{R}^{2}\right):\left.p\right|_{\Gamma_{V}}=\nu\right\} .
$$

The remainder of the paper is dedicated to the study of minimizers of $E_{\varepsilon}$ in $H_{D}^{1}$.

Proposition 2.1. For each fixed $\varepsilon>0$, the functional $E_{\varepsilon}$ attains its minimum.

Proof. Existence of minimizers of $E_{\varepsilon}$ in $H_{D}^{1}$ follows by standard calculus of variations arguments. Let $\left\{p_{j}\right\}$ be a minimizing sequence for $E_{\varepsilon}$ in $H_{D}^{1}$. Taking $p(x)=$ $\left(2 x_{1}-1,0\right)$, we see that $E_{\varepsilon}$ is bounded above in $H_{D}^{1}$. Therefore, $\left\|p_{j}\right\|_{W^{1,2}(\Omega)}<C$, and so there is a subsequence, still labelled $\left\{p_{j}\right\}$, and a $p_{\infty} \in W^{1,2}(\Omega)$, such that

$$
p_{j} \rightarrow p_{\infty} \quad \text { in } W^{1,2}(\Omega) \quad \text { and } \quad p_{j} \rightarrow p_{\infty} \quad \text { in } L^{2}(\Omega) \text { and a.e. in } \Omega .
$$

$L^{2}$-convergence on the boundary follows from the estimate in Theorem 1.5.1.10 in [15], which holds for any Lipschitz domain, and all $0<\delta<1$, giving $\int_{\partial \Omega}\left|p_{j}\right|^{2} \leq$ $C\left(\sqrt{\delta} \int_{\Omega}\left|\nabla p_{j}\right|^{2}+\frac{1}{\sqrt{\delta}} \int_{\Omega}\left|p_{j}\right|^{2}\right)$. Thus, $p_{\infty} \in H_{D}^{1}$ and $E_{\varepsilon}\left(p_{0}\right) \leq \liminf _{j \rightarrow \infty} E_{\varepsilon}\left(p_{j}\right)$.

Lemma 2.2. Let $p_{\varepsilon} \in H_{D}^{1}$ be a critical point of $E_{\varepsilon}$. Then $\left|p_{\varepsilon}\right| \leq 1$ and $\left|\nabla p_{\varepsilon}\right| \leq$ $C / \varepsilon$ for some constant $C$.

Proof. The proof of this lemma follows classical ideas, see [1], albeit modifications needed to treat the non-standard boundary conditions of the problem studied in here.

A critical point of $E_{\varepsilon}(p)$ in $H_{D}^{1}$ satisfies weakly the Euler-Lagrange equations:

$$
\begin{aligned}
& -\Delta p+\frac{|p|^{2}-1}{\varepsilon^{2}} p+\sigma(p-\mathbf{e})=0 \quad \text { in } \Omega \\
& \frac{\partial p}{\partial \nu}+\frac{1}{\varepsilon}(p \cdot \nu) \nu=0 \quad \text { on } \Gamma_{H}, \\
& p=\nu \quad \text { on } \Gamma_{V} .
\end{aligned}
$$

We set $V=|p|^{2}-1$, so that $\nabla V=2 p \cdot \nabla p$, and $\frac{1}{2} \Delta V=p \cdot \Delta p+|\nabla p|^{2}$. Then, by (2.4), we have $\frac{1}{2} \Delta V=p\left(\frac{|p|^{2}-1}{\varepsilon^{2}} p+\sigma(p-\mathbf{e})\right)+|\nabla p|^{2}$, which yields $\frac{1}{2} \Delta V \geq$ $\frac{1}{\varepsilon^{2}}\left[|p|^{2}\left(|p|^{2}-1\right)+\sigma \varepsilon^{2}\left(|p|^{2}-\left|p_{2}\right|\right)\right]$. Multiplying by $V_{+}=\max \{V, 0\}$, we gather $I \equiv$ $\frac{1}{\varepsilon^{2}} \int_{\Omega}\left(|p|^{2}\left(|p|^{2}-1\right)+\sigma \varepsilon^{2}\left(|p|^{2}-\left|p_{2}\right|\right)\right) V_{+} \leq \frac{1}{2} \int_{\Omega} \Delta V \cdot V_{+}$. But, $V_{+} \frac{\partial V}{\partial \nu}=0$ on $\Gamma_{V}$, since $p=\nu$ there, and $V_{+} \frac{\partial V}{\partial \nu}=-\frac{2}{\varepsilon} V_{+}(p \cdot \nu)^{2} \leq 0$ on $\Gamma_{H}$; therefore, we conclude, $I \leq \frac{1}{2} \int_{\partial \Omega} \frac{\partial V}{\partial \nu} V_{+}-\frac{1}{2} \int_{\Omega}\left|\nabla V_{+}\right|^{2} \leq 0$.

On the other hand, $I \geq \frac{1}{\varepsilon^{2}} \int_{\Omega}|p|\left(|p|\left(|p|^{2}-1\right)+\sigma \varepsilon^{2}(|p|-1)\right) V_{+} \geq 0$ since $|p|>1$ where $V_{+} \neq 0$. Therefore $I=0$, hence either $V_{+}=0$ or $/$ and $|p|^{2}\left(|p|^{2}-1\right)+\sigma \varepsilon^{2}\left(|p|^{2}-\right.$ $\left.\left|p_{2}\right|\right)=0$. However, the second case is not possible if $|p|>1$; and, since $V_{+}>0$ implies $|p|>1$, we conclude $V_{+}=0$, which yields $|p|^{2} \leq 1$. 
To prove the gradient estimate, we proceed by contradiction. Suppose there is a sequence $\left\{\varepsilon_{k}\right\} \rightarrow 0$, and $x_{k} \in \bar{\Omega}$ for which $t_{k} \equiv\left|\nabla p_{k}\left(x_{k}\right)\right|=\left\|\nabla p_{k}\right\|_{\infty}$ satisfies $\varepsilon_{k} t_{k} \rightarrow \infty$. Define $v_{k}(x):=p_{k}\left(x_{k}+\frac{x}{t_{k}}\right)$, thanks to the bound proven above, we have $\Delta v_{k}=\frac{1}{t_{k}^{2} \varepsilon_{k}^{2}}\left[\left(\left|v_{k}\right|^{2}-1\right) v_{k}+\sigma \varepsilon_{k}^{2}\left(v_{k}-\mathbf{e}\right)\right] \rightarrow 0$ uniformly. Since the domain of $v_{k}$ is $t_{k}\left(\Omega-x_{k}\right)$, due to the mixed boundary conditions (2.4), we have four possible cases.

Case 1: $t_{k} \operatorname{dist}\left(x_{k} ; \partial \Omega\right) \rightarrow \infty$. In this case, the domain of $v_{k}$ converges to $\mathbb{R}^{2}$. And, we have that the limit $v$ of the $v_{k}$ 's is a bounded harmonic function in $\mathbb{R}^{2}$. By Liouville's theorem, this implies $v$ constant, which in turn gives $\nabla v(x) \equiv 0$, and we have a contraction, since $\left|\nabla v_{k}(0)\right|=1$ for all $k$, hence $|\nabla v(0)|=1$.

Case 2: $t_{k} \operatorname{dist}\left(x_{k} ; \Gamma_{V}\right) \rightarrow \infty$ but $t_{k} \operatorname{dist}\left(x_{k} ; \Gamma_{H}\right)$ is uniformly bounded. In this situation, the domain of $v_{k}$ converges to a half plane. For simplicity, we may assume it to be $\mathbb{R}_{+}^{2}$. Thus, the limit $v$ is an harmonic function on $\mathbb{R}_{+}^{2}$ with homogeneous Neumann boundary condition: $\frac{\partial v_{k}}{\partial \nu}=-\frac{1}{t_{k} \varepsilon_{k}}\left(v_{k} \cdot \nu\right) \nu \rightarrow 0$. But, then the even reflection principle yields a constant solution $v$ in all of $\mathbb{R}^{2}$, and we are back to Case 1 .

Case 3: $t_{k} \operatorname{dist}\left(x_{k} ; \Gamma_{H}\right) \rightarrow \infty$ but $t_{k} \operatorname{dist}\left(x_{k} ; \Gamma_{V}\right)$ is uniformly bounded. We can then assume that the domain of $v_{k}$ converges to the half plane $\mathbb{R}_{+} \times \mathbb{R}$, and that the limit function $v$ is an harmonic function verifying the Dirichlet boundary condition: $v=\nu=-\mathbf{e}_{1}$. Consider $w=v+\mathbf{e}_{1}$, then $w$ is a bounded harmonic function with homogeneous Dirichlet boundary condition. Applying the odd reflection principle to $w$, we obtain that the extension of $w$ is a bounded harmonic function in $\mathbb{R}^{2}$, therefore $w$ is constant, hence $v$ is constant, and as before this leads to a contradiction.

Case 4: $t_{k} \operatorname{dist}\left(x_{k}, \Gamma_{V} \cap \Gamma_{H}\right)$ is uniformly bounded. We can assume that the domain of $v_{k}$ converges to the first quadrant, $\mathbb{R}_{+} \times \mathbb{R}_{+}$, with Dirichlet boundary condition, $v=\nu=-\mathbf{e}_{1}$ on $\{0\} \times \mathbb{R}_{+}$, and homogeneous Neumann boundary condition on $\mathbb{R}_{+} \times\{0\}$. By applying the even reflection principle along the positive $x$-axis, we obtain $v$ iconstant following the argument in Case 3 , hence we have a contradiction.

We conclude that there exists a $C$ such that $\left|\nabla p_{\varepsilon}\right| \leq C / \varepsilon$.

For global minimizers, in the presence of an applied electric field, it is easy to see that the $y$-component of $p_{\varepsilon}$ follows the field. In the absence of an applied field, i.e. when $\sigma=0$, we instead expect global minimizers to have $y$-component either always positive or always negative, in accordance with the expected bistability of the phase.

LEMMA 2.3. Let $p_{\varepsilon}=\left(p_{1}, p_{2}\right)$ be a global minimizer for $E_{\varepsilon}(p)$ in $H_{1}^{D}$. We have that if $\sigma \neq 0$ then $\sigma_{s} p_{2} \geq 0$; while if $\sigma=0$ then $p_{2}$ does not change sign in $\Omega$.

Proof. Assume $\sigma \neq 0$. Given a global minimizer $p_{\varepsilon}=\left(p_{1}, p_{2}\right)$, we consider $\tilde{p}_{\varepsilon}=\left(p_{1}, \sigma_{s}\left|p_{2}\right|\right)$, and compute its energy: $E_{\varepsilon}\left(\tilde{p}_{\varepsilon}\right) \leq E_{\varepsilon}\left(p_{\varepsilon}\right)-2 \sigma \int_{\Omega}\left(\left|p_{2}\right|-\sigma_{s} p_{2}\right) d x$; and, since $p_{\varepsilon}$ is a global minimizer, this implies $\sigma_{s} p_{2}=\left|p_{2}\right| \geq 0$ a.e. in $\Omega$.

If $\sigma=0$, and $p_{\varepsilon}=\left(p_{1}, p_{2}\right)$ is a global minimizer, as above we have that $\tilde{p}_{\varepsilon}=$ $\left(p_{1},\left|p_{2}\right|\right)$ is also a global minimizer, since $E_{\varepsilon}\left(\tilde{p}_{\varepsilon}\right)=E_{\varepsilon}\left(p_{\varepsilon}\right)$. In particular, both $p_{\varepsilon}$ and $\tilde{p}_{\varepsilon}$ verify $(2.4)$. Set $\omega=\frac{1}{2}\left(p_{2}-\left|p_{2}\right|\right) \equiv \min \left\{0, p_{2}\right\} \leq 0$, then $\omega \in H^{1}(\Omega ; \mathbb{R})$; moreover, because $\left|p_{\varepsilon}\right|=\left|\tilde{p}_{\varepsilon}\right|$, it holds

$$
\begin{aligned}
& \Delta \omega=\frac{|p|^{2}-1}{\varepsilon^{2}} \omega \geq 0 \quad \text { in } \Omega, \\
& \frac{\partial \omega}{\partial \nu}+\frac{1}{\varepsilon} \omega=0 \quad \text { on } \Gamma_{H}, \\
& \omega=0 \quad \text { on } \Gamma_{V} .
\end{aligned}
$$

The right hand-side of the first equation in (2.5) is bounded in $\Omega$, hence we can apply Theorem 8.22 in [13] to obtain that $\omega$ is continuous in $\Omega$. 
Assume that there exists $x_{0} \in \Omega$ for which $\omega\left(x_{0}\right)=0$, pick $R>0$ small enough so to have $B_{R}\left(x_{0}\right) \Subset \Omega$; given that $\omega \leq 0$ by definition, this implies $\sup _{B_{R}\left(x_{0}\right)} \omega=\sup _{\Omega} \omega$. Therefore, by Theorem 8.19 in [13], we gather that $\omega \equiv 0$, or in other words $p_{2} \geq 0$ in $\Omega$. If such $x_{0}$ does not exist, then $\omega<0$ in $\Omega$, and we conclude $p_{2} \leq 0$ in $\Omega$.

REMARK 2.1. A solution in $H^{1}\left(\Omega ; \mathbb{R}^{2}\right)$ of (2.4) belongs to $C^{\infty}\left(\Omega ; \mathbb{R}^{2}\right) \cap C^{0}\left(\bar{\Omega} ; \mathbb{R}^{2}\right)$ by the classical regularity theory for elliptic problems (for boundary regularity of mixed boundary value problem see [20], and reference thereafter).

In the remainder of the paper, we assume $\sigma=\mathcal{O}(1)$ for $\varepsilon \rightarrow 0$; and for $a \in \mathbb{R}^{2}$, and $R>0$ we adopt the following notation:

1. $\Omega_{R}(a)=\Omega \cap B_{R}(a), \Gamma_{R}^{V}(a)=\Gamma_{V} \cap B_{R}(a)$, and $\Gamma_{R}^{H}(a)=\Gamma_{H} \cap B_{R}(a)$;

2. $\Gamma_{H}^{T}$ and $\Gamma_{H}^{B}$ are the top, and bottom portions of $\Gamma_{H}$, respectively;

3. Given a set $G$ with $G \cap \Omega \neq \emptyset, E_{\varepsilon}\left(p_{\varepsilon} ; G\right)$ is the restriction of $E_{\varepsilon}\left(p_{\varepsilon}\right)$ to $G$;

4. $A_{r, R}(a)=\Omega_{R}(a) \backslash \Omega_{r}(a)$ for $0<r<R$;

5. $\mathcal{V}$ is the set of the four corners of $\Omega$;

6. $\beta$ and $\gamma$ denote any two numbers such that $3 / 4 \leq \beta<\gamma<1$.

3. Basic estimates. To show convergence of global minimizers, we need upper and lower bound estimates for the energy of near defects, obtained following ideas first introduced in the classical work of Bethuel, Brezis, and Hélein [5], for the Ginzburg-Landau energy of superconductivity. The task here is to refine and adjust this approach to accommodate the new specific properties of our model: a Lipschitz domain, and a mixed of strong and weak anchoring conditions. The main references we follow are the works of Alama et al. [1] and [2], Struwe [32], and Moser [23].

3.1. Energy upper bound. In this section, we obtain an upper bound for the energy $E_{\varepsilon}$ of global minimizers, which combined with the lower bound estimates for the energy of near defects derived in Section 3.3 will show that having internal near vortices is too energetically demanding compared to having near boundary vortices.

Proposition 3.1. There is $C>0$, and $\varepsilon_{0}>0$ such that for $\varepsilon<\varepsilon_{0}$ any global minimizer of $E_{\varepsilon}$ in $H_{D}^{1}$ satisfies $E_{\varepsilon}\left(p_{\varepsilon}\right) \leq \pi|\ln \varepsilon|+C(1+\sigma)$.

Proof. Our numerical results in Section 5 suggest that stable minimizers have two off-domain symmetric boundary vortices at distance $\varepsilon$ from an horizontal side. Thus, we pick $a_{1}=(1 / 2,0), a_{2}=(1 / 2,1)$, and $R>0$ so that $\Omega_{3 R}\left(a_{i}\right)$ are disjoint half discs, for $i=1,2$; and, following [23], we define locally an $\mathbb{S}^{1}$ valued map $p^{\varepsilon}$, which has exactly two boundary vortices located at distance $\varepsilon$ from $a_{1}$ and $a_{2}$. That is, we set $p^{\varepsilon}(x)=\left(x-a_{1 \varepsilon}\right) /\left|x-a_{1 \varepsilon}\right|$ if $x \in B_{2 R}\left(a_{1}\right)$, and $p^{\varepsilon}(x)=\left(\overline{x-a_{2 \varepsilon}}\right) /\left|x-a_{2 \varepsilon}\right|$ if $x \in B_{2 R}\left(a_{2}\right)$. In here $a_{i \varepsilon}=a_{i}+\varepsilon \nu\left(a_{i}\right)$ for $i=1,2$. Pick $\varepsilon_{0}>0$ small enough depending on $R$, we estimate the contribution to the energy density by direct computations:

$$
\begin{aligned}
& \frac{1}{2} \int_{\Omega_{R}\left(a_{1}\right)}\left|\nabla p^{\varepsilon}\right|^{2} d x \leq \frac{1}{2} \int_{B_{2 R}^{+}\left(a_{1 \varepsilon}\right) \backslash B_{\varepsilon}^{+}\left(a_{1 \varepsilon}\right)}\left|\nabla p^{\varepsilon}\right|^{2} d x \leq \frac{\pi}{2}(\ln (2 R)-\ln \varepsilon) ; \\
& \frac{1}{2} \int_{\Omega_{2 R}\left(a_{1}\right) \backslash \Omega_{R}\left(a_{1}\right)}\left|\nabla p^{\varepsilon}\right|^{2} d x \leq C ; \text { and } \int_{\Gamma_{2 R}^{H}\left(a_{1}\right)}\left(p^{\varepsilon} \cdot \nu\right)^{2} d x \leq \int_{-2 R}^{2 R} \frac{\varepsilon^{2}}{x^{2}+\varepsilon^{2}} d x \leq \pi \varepsilon ;
\end{aligned}
$$

where, for $s>0, B_{s}^{+}\left(a_{1 \varepsilon}\right)$ denotes the upper-half of the ball of radius $r$ and center $a_{1 \varepsilon}$. We extend $p^{\varepsilon}$ to the whole domain, in such a way that its energy contributions will be of lower order. Specifically, along the horizontal boundary we define $p^{\varepsilon}=-\mathbf{e}_{1}$ if $x \leq \frac{1}{2}-3 R$, and $p^{\varepsilon}=\mathbf{e}_{1}$ if $x \geq \frac{1}{2}+3 R$. On the rest of the horizontal boundary, $\Gamma_{3 R}^{H}\left(a_{i}\right) \backslash \Gamma_{2 R}^{H}\left(a_{i}\right)$, we continuously connect the map at $\left|x-a_{i}\right|=2 R$ and $\left|x-a_{i}\right|=3 R$. 
Then $p_{2}^{\varepsilon} \leq C \varepsilon$ there, so that $\int_{\Gamma_{3 R}^{H}\left(a_{i}\right) \backslash \Gamma_{2 R}^{H}\left(a_{i}\right)}\left(p^{\varepsilon} \cdot \nu\right)^{2} \leq C \varepsilon^{2}$. Proceeding similarly on $\Omega_{2 R}\left(a_{2}\right)$, we arrive to $E_{\varepsilon}\left(p^{\varepsilon} ; \Omega_{2 R}\left(a_{1}\right) \cup \Omega_{2 R}\left(a_{2}\right)\right) \leq \pi|\ln \varepsilon|+C(1+\sigma)$.

To extend $p_{\varepsilon}$ to the region $\tilde{\Omega}=\Omega \backslash \cup_{i=1}^{2} \Omega_{2 R}\left(a_{i}\right)$, we consider the function $g^{\varepsilon}(x)=$ $\nu$ on $\Gamma_{V}, g^{\varepsilon}(x)=p_{\varepsilon}$ on $\partial \tilde{\Omega} \backslash \Gamma_{V}$, and notice that $\operatorname{deg}\left(g^{\varepsilon} ; \partial \tilde{\Omega}\right)=0$; then as in [1], we can define $p_{\varepsilon}$ on $\tilde{\Omega}$ to be the $\mathbb{S}^{1}$-valued harmonic extension of $g^{\varepsilon}$.

3.2. $\eta$-compactness. A key ingredient in the derivation of an energy lower bound is the so-called $\eta$-compactness (Proposition 3.3), which is essential in proving a bound for the number of near defects allowed in a global minimizer (Lemma 4.1).

Define $F\left(r ; x_{0}, p, \varepsilon\right)$, which we will use to bound the penalty terms of $E_{\varepsilon}(p)$, as

$$
\begin{gathered}
F(r) \equiv F\left(r ; x_{0}, p, \varepsilon\right) r\left[\int_{\partial B_{r}\left(x_{0}\right) \cap \Omega}\left\{|\nabla p|^{2}+\frac{1}{2 \varepsilon^{2}}\left(1-|p|^{2}\right)^{2}+\sigma|\mathbf{e}-p|^{2}\right\}\right. \\
\left.+\frac{1}{\varepsilon} \sum_{x \in \partial B_{r}\left(x_{0}\right) \cap \Gamma_{H}}(p \cdot \nu)^{2}\right] .
\end{gathered}
$$

LEMma 3.2. There are $C, r_{0}>0$ such that for $\varepsilon<1, x_{0} \in \Gamma, r<r_{0}$ and for a critical point $p_{\varepsilon}$ of $E_{\varepsilon}$, we have

$$
\int_{\Omega_{r}\left(x_{0}\right)}\left[\frac{1}{2 \varepsilon^{2}}\left(1-\left|p_{\varepsilon}\right|^{2}\right)^{2}+\sigma\left|\mathbf{e}-p_{\varepsilon}\right|^{2}\right]+\frac{1}{2 \varepsilon} \int_{\Gamma_{r}^{H}\left(x_{0}\right)}\left(p_{\varepsilon} \cdot \nu\right)^{2} d s \leq \frac{1}{2} F(r)+\sigma r^{2}
$$

Proof. Consider the following Pohozaev identity, which we derive by multiplying the Euler-Lagrange equations $(2.4)$ by $X \cdot \nabla p$ (see [34, 1]):

$$
\int_{\partial \Omega_{r}}\left[-\frac{\partial p}{\partial \nu} X \cdot \nabla p+\frac{1}{2}|\nabla p|^{2}(X \cdot \nu)+\left\{\frac{1}{4 \varepsilon^{2}}\left(1-|p|^{2}\right)^{2}+\frac{\sigma}{2}|\mathbf{e}-p|^{2}\right\} X \cdot \nu\right] d x
$$

$$
=\int_{\Omega_{r}}\left[\left\{\frac{1}{4 \varepsilon^{2}}\left(1-|p|^{2}\right)^{2}+\frac{\sigma}{2}|\mathbf{e}-p|^{2}\right\} \nabla \cdot X+\frac{1}{2}|\nabla p|^{2}(\nabla \cdot X)-\nabla p D X \nabla p\right] d x,
$$

for any $X \in C^{\infty}\left(\Omega ; \mathbb{R}^{2}\right)$, where $D X$ denotes the matrix of partial derivatives of $X$.

Let $r_{0}$ small enough, so that if $r<r_{0}$ and $\partial B_{r}\left(x_{0}\right) \cap \Gamma_{H} \neq \emptyset$, then $\partial B_{r}\left(x_{0}\right) \cap \partial \Omega$ consists of either two points on $\Gamma_{H}$ or one point on $\Gamma_{H}$ if $B_{r}\left(x_{0}\right) \cap \Gamma_{V} \neq \emptyset$. Given $x_{0} \in \Gamma_{H}$, and $r<r_{0}$, suppose that $B_{r}\left(x_{0}\right) \cap \Gamma_{V}=\emptyset$. Take $X=x-x_{0}$, then $X \cdot \nu=0$ on $\Gamma_{H}\left(x_{0}\right)$ and $X \cdot \nu=r$ on $\partial \Omega_{r}\left(x_{0}\right) \cap \Omega$. Using equation (2.4), we can find an upper bound for the left hand side $L H S$ of (3.2). We start from

$$
\begin{aligned}
& \int_{\Gamma_{r}^{H}\left(x_{0}\right)}-\frac{\partial p}{\partial \nu} X \cdot \nabla p d s=\frac{1}{\varepsilon} \int_{\Gamma_{r}^{H}\left(x_{0}\right)} p_{2}\left(x_{1}-\left(x_{0}\right)_{1}\right)\left(p_{2}\right)_{x_{1}} d s \\
& =\frac{1}{2 \varepsilon} \int_{\Gamma_{r}^{H}\left(x_{0}\right)}\left(p_{2}^{2}\right)_{x_{1}}\left(x_{1}-\left(x_{0}\right)_{1}\right) d s=\frac{1}{2 \varepsilon}\left(r \sum_{x \in \partial \Gamma_{r}^{H}\left(x_{0}\right)} p_{2}^{2}(x)-\int_{\Gamma_{r}^{H}\left(x_{0}\right)} p_{2}^{2} d s\right),
\end{aligned}
$$

and, noticing that $X \cdot \tau=0$ and $X \cdot \nu=r$ on $\partial \Omega_{r}\left(x_{0}\right) \cap \Omega$, we gather

$$
\int_{\partial \Omega_{r}\left(x_{0}\right) \cap \Omega}\left(-\frac{\partial p}{\partial \nu} X \cdot \nabla p+\frac{1}{2}|\nabla p|^{2}(X \cdot \nu)\right) d s \leq \frac{r}{2} \int_{\partial \Omega_{r}\left(x_{0}\right) \cap \Omega}|\nabla p|^{2} .
$$

Therefore, we conclude 


$$
\begin{aligned}
L H S & \leq \frac{1}{2 \varepsilon}\left(r \sum_{\partial \Gamma_{r}^{H}\left(x_{0}\right)} p_{2}^{2}(x)-\int_{\Gamma_{r}^{H}\left(x_{0}\right)} p_{2}^{2} d s\right)+\frac{r}{2} \int_{\partial \Omega_{r}\left(x_{0}\right) \cap \Omega}|\nabla p|^{2} d s \\
& +r \int_{\partial \Omega_{r}\left(x_{0}\right) \cap \Omega}\left(\frac{1}{4 \varepsilon^{2}}\left(1-|p|^{2}\right)^{2}+\frac{1}{2} \sigma|\mathbf{e}-p|^{2}\right) d s .
\end{aligned}
$$

But, $X=x-x_{0}$ implies $D X=I d$, and $\nabla \cdot X=2$, so the last two terms of the right hand side of (3.2) vanish. Inequality (3.1) is then a consequence of (3.2) and (3.3).

If instead, $B_{r}\left(x_{0}\right) \cap \Gamma_{V} \neq \emptyset$, we rewrite $L H S$ in three parts:

$$
L H S:=\int_{\partial \Omega_{r}\left(x_{0}\right)} l(s) d s=\int_{\Gamma_{r}^{V}\left(x_{0}\right)} l(s) d s+\int_{\Gamma_{r}^{H}\left(x_{0}\right)} l(s) d s+\int_{\partial \Omega_{r}\left(x_{0}\right) \cap \Omega} l(s) d s .
$$

The last two integrals can be estimated as above. For the first integral, we instead notice that $p=\nu$ on $\Gamma_{r}^{V}\left(x_{0}\right)$ gives $|p|=1$ and $\frac{\partial p}{\partial \tau}=0$ and $X \cdot \nu>0$ on $\Gamma_{r}^{V}\left(x_{0}\right)$, so that

$$
\begin{aligned}
\int_{\Gamma_{r}^{V}\left(x_{0}\right)} l(s) d s=\int_{\Gamma_{r}^{V}\left(x_{0}\right)}\left(-\frac{\partial p}{\partial \nu} X \cdot\left(\frac{\partial p}{\partial \nu} \nu+\frac{\partial p}{\partial \tau} \tau\right)+\frac{1}{2}|\nabla p|^{2} X \cdot \nu\right. \\
\left.+\frac{\sigma}{2}|\mathbf{e}-\nu|^{2}(X \cdot \nu)\right)=\int_{\Gamma_{r}^{V}\left(x_{0}\right)}\left(-\frac{\partial p}{\partial \nu} \frac{\partial p}{\partial \nu} X \cdot \nu+\frac{1}{2}|\nabla p|^{2} X \cdot \nu+\sigma(X \cdot \nu)\right) \\
=\int_{\Gamma_{r}^{V}\left(x_{0}\right)}\left(-\frac{1}{2}|\nabla p|^{2} X \cdot \nu+\sigma(X \cdot \nu)\right) \leq \sigma r^{2}
\end{aligned}
$$

which yields (3.1). The case $x_{0} \in \Gamma_{V}$ can be treated similarly.

The $\eta$-compactness result stated in the next proposition is proven using Lemma 3.2, and proceeding with suitable adjustments as in Proposition 4.1 of [1].

Proposition 3.3. There exist $\eta, C, \varepsilon_{0}>0$ such that for every $x_{0} \in \bar{\Omega}$, and $\varepsilon<\varepsilon_{0}$, and every critical point $p_{\varepsilon}$ of $E_{\varepsilon}$ with $E_{\varepsilon}\left(p_{\varepsilon} ; \Omega_{\varepsilon^{\beta}}\left(x_{0}\right)\right) \leq \eta|\ln \varepsilon|$ it holds

$$
\begin{aligned}
& \left|p_{\varepsilon}\right| \geq \frac{1}{2} \quad \text { in } \Omega_{\varepsilon^{\gamma}}\left(x_{0}\right), \\
& \left|p_{\varepsilon} \cdot \nu\right| \leq \frac{1}{4} \quad \text { on } \Gamma_{\varepsilon^{\gamma}}^{H}\left(x_{0}\right), \\
& \int_{\Omega_{\varepsilon} \gamma\left(x_{0}\right)}\left(\frac{1}{2 \varepsilon^{2}}\left(1-\left|p_{\varepsilon}\right|^{2}\right)^{2}+\sigma\left|\mathbf{e}-p_{\varepsilon}\right|^{2}\right) d x+\frac{1}{\varepsilon} \int_{\Gamma_{\varepsilon \gamma}^{H}\left(x_{0}\right)}\left(p_{\varepsilon} \cdot \nu\right)^{2} d s \leq C \eta .
\end{aligned}
$$

Proof. If $x_{0} \in \Omega \cup \Gamma_{V}$ the proposition is proven as in [32]. Assume $x_{0} \in \Gamma_{H}$. As in [1], we note that $\eta|\ln \varepsilon| \geq E_{\varepsilon}\left(p_{\varepsilon} ; \Omega_{\varepsilon^{\beta}} \backslash \Omega_{\varepsilon^{\gamma}}\right)=\int_{\varepsilon^{\gamma}}^{\varepsilon^{\beta}} \frac{F(r)}{r} d r$, which implies

$$
F\left(r_{\varepsilon}\right)(\gamma-\beta) \leq \eta,
$$

for some $r_{\varepsilon} \in\left(\varepsilon^{\gamma}, \varepsilon^{\beta}\right)$. Define $\widetilde{E_{\varepsilon}}\left(p_{\varepsilon} ; \Omega_{\varepsilon^{\gamma}}\right)=E_{\varepsilon}\left(p_{\varepsilon} ; \Omega_{\varepsilon^{\gamma}}\right)-\frac{1}{2} \int_{\Omega_{\varepsilon} \gamma}\left|\nabla p_{\varepsilon}\right|^{2}$, and use Lemma 3.2, to obtain $\widetilde{E_{\varepsilon}}\left(p_{\varepsilon} ; \Omega_{\varepsilon \gamma}\right) \leq \widetilde{E_{\varepsilon}}\left(p_{\varepsilon} ; \Omega_{r_{\varepsilon}}\right) \leq \frac{1}{2} F\left(r_{\varepsilon}\right)+\sigma r_{\varepsilon}^{2} \leq C\left[\eta+\sigma \varepsilon^{2 \beta}\right] \leq C \eta$.

Inequality (3.4), is proven by contradiction. Assume there exists $x_{1} \in \Omega_{\varepsilon \gamma}\left(x_{0}\right)$ such that $\left|p_{\varepsilon}\left(x_{1}\right)\right|<\frac{1}{2}$. Since Lemma 2.2 implies $\left|\nabla p_{\varepsilon}\right| \leq C / \varepsilon$, this gives $\left|p_{\varepsilon}(x)\right|<3 / 4$ in $B_{\frac{\varepsilon}{4 C}}\left(x_{1}\right)$, which together with (3.6) yields $C \eta>\frac{1}{4 \varepsilon^{2}} \int_{\Omega_{\varepsilon}\left(x_{0}\right)}\left(1-\left|p_{\varepsilon}\right|^{2}\right)^{2} \geq \frac{49 \pi}{2^{14} C^{2}}$ for sufficiently small $\varepsilon_{0}$, which results in a contradiction if $\eta$ is sufficiently small. 
We are left to show (3.5). We pick $\epsilon_{0}$ small enough, so that either $\Omega_{r}\left(x_{0}\right) \cap \Gamma_{H}^{T}=\emptyset$ or $\Omega_{r}\left(x_{0}\right) \cap \Gamma_{H}^{B}=\emptyset$, (note that $r=r_{\varepsilon}$ in (3.7)), and apply Pohozaev identity (3.2), with $X=-r \mathbf{e}_{2}$ if $\Omega_{r}\left(x_{0}\right) \cap \Gamma_{H}^{T}=\emptyset$, and $X=r \mathbf{e}_{2}$ if $\Omega_{r}\left(x_{0}\right) \cap \Gamma_{H}^{B}=\emptyset$. With these choices, except at the two points where $\partial B_{r}\left(x_{0}\right)$ meets $\partial \Omega$, we have:

$$
\begin{array}{ccc}
X \cdot \nu(x)=0 & \text { if } x \in \partial \Omega_{r}\left(x_{0}\right) \cap \Gamma_{V}, \\
|X \cdot \nu(x)| \leq r \text { and }|X \cdot \tau(x)| \leq r & \text { if } x \in \partial \Omega_{r}\left(x_{0}\right) \backslash\left(\Gamma_{V} \cup \Gamma_{H}\right), \\
X \cdot \nu(x)=r \text { and } X \cdot \tau(x)=0 & \text { if } x \in \partial \Omega_{r}\left(x_{0}\right) \cap \Gamma_{H} .
\end{array}
$$

On the other hand, we have $-\frac{\partial p}{\partial \nu} X \cdot \nabla p=-X \cdot \nu\left|\frac{\partial p}{\partial \nu}\right|^{2}-\frac{\partial p}{\partial \nu} \cdot \frac{\partial p}{\partial \tau} X \cdot \tau$; and $|\nabla p|^{2}=\left|\frac{\partial p}{\partial \nu}\right|^{2}+\left|\frac{\partial p}{\partial \tau}\right|^{2}$. Thus, (3.8), (3.10), and the fact that $\frac{\partial p}{\partial \tau}=0$ on $\Gamma_{V}$, imply

$$
\begin{array}{ll}
-\frac{\partial p}{\partial \nu} X \cdot \nabla p+\frac{|\nabla p|^{2}}{2}(X \cdot \nu)=0 & \text { on } \partial \Omega_{r}\left(x_{0}\right) \cap \Gamma_{V}, \\
-\frac{\partial p}{\partial \nu} X \cdot \nabla p+\frac{|\nabla p|^{2}}{2}(X \cdot \nu)=\frac{1}{2}\left|\frac{\partial p}{\partial \tau}\right|^{2}(X \cdot \nu) &
\end{array}
$$

$$
\begin{array}{crl}
-\frac{1}{2}\left|\frac{\partial p}{\partial \nu}\right|^{2}(X \cdot \nu)-\frac{\partial p}{\partial \nu} \cdot \frac{\partial p}{\partial \tau} X \cdot \tau & \text { on } \partial \Omega_{r}\left(x_{0}\right) \backslash\left(\Gamma_{V} \cup \Gamma_{H}\right), \\
-\frac{\partial p}{\partial \nu} X \cdot \nabla p+\frac{|\nabla p|^{2}}{2}(X \cdot \nu)=-\frac{r}{2}\left|\frac{\partial p}{\partial \nu}\right|^{2}+\frac{r}{2}\left|\frac{\partial p}{\partial \tau}\right|^{2} & \text { on } \partial \Omega_{r}\left(x_{0}\right) \cap \Gamma_{H} .
\end{array}
$$

Going back to the Pohozaev identity, we notice that $X$ constant results in the right hand side to be zero, that is we have

$$
\int_{\partial \Omega_{r}}\left[-\frac{\partial p}{\partial \nu} X \cdot \nabla p+\frac{1}{2}|\nabla p|^{2}(X \cdot \nu)+\left\{\frac{1}{4 \varepsilon^{2}}\left(1-|p|^{2}\right)^{2}+\frac{\sigma}{2}|\mathbf{e}-p|^{2}\right\} X \cdot \nu\right] d x=0
$$

If we use equations (3.11), (3.8) and (3.10) in (3.12), we can further simplify:

$$
\begin{aligned}
& \frac{r}{2} \int_{\partial \Omega_{r} \cap \Gamma_{H}}\left|\frac{\partial p}{\partial \tau}\right|^{2} d x=\int_{\partial \Omega_{r} \cap \Gamma_{H}}\left[\frac{r}{2}\left|\frac{\partial p}{\partial \nu}\right|^{2}-r\left\{\frac{1}{4 \varepsilon^{2}}\left(1-|p|^{2}\right)^{2}+\frac{\sigma}{2}|\mathbf{e}-p|^{2}\right\}\right] d x \\
& -\int_{\partial \Omega_{r} \backslash\left(\Gamma_{V} \cup \Gamma_{H}\right)}\left[\frac{1}{2}\left[-\left|\frac{\partial p}{\partial \nu}\right|^{2}+\left|\frac{\partial p}{\partial \tau}\right|^{2}\right](X \cdot \nu)-\frac{\partial p}{\partial \nu} \cdot \frac{\partial p}{\partial \tau} X \cdot \tau\right. \\
& \left.\quad+\left\{\frac{1}{4 \varepsilon^{2}}\left(1-|p|^{2}\right)^{2}+\frac{\sigma}{2}|\mathbf{e}-p|^{2}\right\} X \cdot \nu\right] d x .
\end{aligned}
$$

On $\partial \Omega_{r} \backslash\left(\Gamma_{V} \cup \Gamma_{H}\right)$, it holds $\left|\frac{1}{2}\left[-\left|\frac{\partial p}{\partial \nu}\right|^{2}+\left|\frac{\partial p}{\partial \tau}\right|^{2}\right](X \cdot \nu)-\frac{\partial p}{\partial \nu} \cdot \frac{\partial p}{\partial \tau} X \cdot \tau\right| \leq$ $r\left(\left|\frac{\partial p}{\partial \nu}\right|^{2}+\left|\frac{\partial p}{\partial \tau}\right|^{2}\right)$, thanks to (3.9). From this, using (3.9), and (3.13) we gather

$$
\begin{aligned}
& \frac{r}{2} \int_{\partial \Omega_{r} \cap \Gamma_{H}}\left|\frac{\partial p}{\partial \tau}\right|^{2} d x \leq \int_{\partial \Omega_{r} \cap \Gamma_{H}} \frac{r}{2}\left|\frac{\partial p}{\partial \nu}\right|^{2} d x \\
& \quad+\quad r \int_{\partial \Omega_{r} \backslash\left(\Gamma_{V} \cup \Gamma_{H}\right)}\left[|\nabla p|^{2}+\left\{\frac{1}{4 \varepsilon^{2}}\left(1-|p|^{2}\right)^{2}+\frac{\sigma}{2}|\mathbf{e}-p|^{2}\right\}\right] d x .
\end{aligned}
$$


Dividing by $r$, and recalling the definition of $F(r)$, we see that

$$
\frac{1}{2} \int_{\partial \Omega_{r} \cap \Gamma_{H}}\left|\frac{\partial p}{\partial \tau}\right|^{2} d x \leq \int_{\partial \Omega_{r} \cap \Gamma_{H}} \frac{1}{2}\left|\frac{\partial p}{\partial \nu}\right|^{2} d x+\frac{F(r)}{r} .
$$

And, combining it with (2.4), (3.7) and (3.6), we conclude

$$
\int_{\partial \Omega_{r} \cap \Gamma_{H}}\left|\frac{\partial p}{\partial \tau}\right|^{2} d x \leq \int_{\partial \Omega_{r} \cap \Gamma_{H}} \frac{1}{\varepsilon^{2}}(p \cdot \nu)^{2} d x+C \frac{\eta}{r} \leq C \eta\left(\frac{1}{\varepsilon}+\frac{1}{\varepsilon^{\gamma}}\right) \leq \frac{C}{\varepsilon}
$$

Following $[1,23]$, since the bound in (3.14) does not depend on $x_{0}$, by Sobolev embedding theorems there exists $C_{1}$, independent of $x_{0}$, verifying

$$
|p(x)-p(y)| \leq C_{1} \sqrt{|x-y|} \varepsilon^{-1 / 2} \quad \text { for } x, y \in \Gamma_{H},
$$

then a similar proof to the one used to obtain (3.4) can be applied: If there was an $x_{1} \in \Gamma_{\varepsilon^{\gamma}}^{H}\left(x_{0}\right)$ with $\left|(p \cdot \nu)\left(x_{1}\right)\right|>\frac{1}{4}$, we could pick $2 \rho=\varepsilon /\left(8 C_{1}\right)^{2}$, so that

$$
\begin{aligned}
& |(p \cdot \nu)(x)| \geq\left|(p \cdot \nu)\left(x_{1}\right)\right|-\left|(p \cdot \nu)(x)-(p \cdot \nu)\left(x_{1}\right)\right| \geq \frac{1}{4}-\left|p(x)-p\left(x_{1}\right)\right| \\
& \quad \geq \frac{1}{4}-C_{1} \sqrt{\left|x-x_{1}\right|} \varepsilon^{-1 / 2} \geq \frac{1}{4}-C_{1} \sqrt{2 \rho} \varepsilon^{-1 / 2} \geq \frac{1}{4}-C_{1} \frac{\varepsilon^{1 / 2}}{8 C_{1}} \varepsilon^{-1 / 2}=\frac{1}{8},
\end{aligned}
$$

for $x \in B_{\rho}\left(x_{1}\right) \cap \Gamma_{H}$. Then, by (3.6), and $\epsilon^{\gamma}>\epsilon$ for $\epsilon<1$, we would have $C \eta \geq$ $\frac{1}{\varepsilon} \int_{\Gamma_{\varepsilon \gamma}^{H}\left(x_{0}\right)}\left(p_{\varepsilon} \cdot \nu\right)^{2} d s>\frac{1}{\varepsilon} \frac{1}{64} \frac{\varepsilon}{\left(8 C_{1}\right)^{2}}=\frac{1}{64} \frac{1}{\left(8 C_{1}\right)^{2}}$, which for $\eta$ small enough would lead to a contradiction. Note that for $\varepsilon_{0}$ small enough, we should have that the length of $B_{\rho}\left(x_{1}\right) \cap \Gamma_{H}$ is at least $\rho$, for any $x_{1} \in \Gamma_{\varepsilon^{\gamma}}^{H}\left(x_{0}\right)$, since all the constants $C$ and $C_{1}$ above are independent of $\epsilon$ and $x_{0}$.

3.3. Energy cost of a vortex. In this section we derive estimates needed to evaluate the energy cost of vortices; the cost will vary depending on the vortex location (see Lemma 3.4 and Proposition 3.5), with lowest energy achieved by vortices on the horizontal boundary. In order to establish comparison arguments as in [32], we need a lower bound of the energy in an annular region around a near defect.

For $x_{0} \in \Gamma$ and $R<1 / 2, \partial B_{R}\left(x_{0}\right) \cap \Gamma$ consists of two points, which are either both on only one of the four sides of $\Omega$, or one is on $\Gamma_{H}$ and the other on $\Gamma_{V}$. Additionally, $\partial A_{r, R}\left(x_{0}\right) \cap \partial \Omega$ consists of two connected components, which we denote by $\Gamma_{r, R}^{ \pm}$.

Following [23], one sees that on $\Gamma_{H}$ the inequality $|p \cdot \nu| \leq 1 / 4$ implies $|p \cdot \tau| \geq \sqrt{3} / 4$. So, if $|p \cdot \nu| \leq 1 / 4$ then $p \cdot \tau$ does not change its sign on a connected component. Note that $\tau=\left(-\nu_{2}, \nu_{1}\right)$ when $\nu=\left(\nu_{1}, \nu_{2}\right)$, hence $p \cdot \tau=p_{1}$ on $\Gamma_{H}^{B}$, and $p \cdot \tau=-p_{1}$ on $\Gamma_{H}^{T}$.

As in $[23,1]$, we will need to represent $p$ in polar coordinates to estimate the energy around a near defect, and this representation will depend on its location.

Assume $R<1 / 2, \frac{1}{2} \leq|p| \leq 1$ on $A_{r, R}\left(x_{0}\right)$, and $|p \cdot \nu| \leq \frac{1}{4}$ on $\Gamma_{r, R}^{ \pm} \cap \Gamma_{H}$. And, recall that $p \cdot \nu=1$ on $\Gamma_{V}$. We begin with the following three cases.

Case 1: $x_{0} \in \Gamma_{H}, B_{R}\left(x_{0}\right) \cap \Gamma_{V}=\emptyset$. If $p \cdot \tau$ does not change sign on $\Gamma_{r, R}^{ \pm}$, we extend $p$ to $\tilde{p}$ on all of $B_{R}\left(x_{0}\right) \cap \Gamma_{H}$, so that $\tilde{p}$ is smooth as $p,|\tilde{p}| \geq 1 / 2$, and $|\tilde{p} \cdot \nu| \leq 1 / 4$ on $B_{R}\left(x_{0}\right) \cap \Gamma_{H}$. Set $\tilde{p}=p$ on the rest of $\partial \Omega_{R}\left(x_{0}\right)$, and define $\tilde{d}=\operatorname{deg}\left(\frac{\tilde{p}}{\mid \tilde{p}}, \partial \Omega_{R}\left(x_{0}\right)\right)$. Letting $\rho=\left|x-x_{0}\right|$, and with $\theta$ measured with respect to the positively oriented horizontal line segment $\Gamma_{r, R}^{+}$, we have the representation: $p=\zeta(\rho, \theta) e^{i(2 \tilde{d} \theta+\phi(\rho, \theta))}$, where $\phi$ is a smooth single valued function in $A_{r, R}\left(x_{0}\right)$, and $x=x_{0}+\rho e^{i \theta}$. 
If $p \cdot \tau$ does change its sign, assuming, without loss of generality, $x_{0}=(0,0)$, $p \cdot \tau>0$ on $\Gamma_{r, R}^{+}$, and $p \cdot \tau<0$ on $\Gamma_{r, R}^{-}$; then, $p e^{-i \theta} \cdot \tau$ does not change its sign on $\Gamma_{r, R}^{ \pm}$. And, we can extend $p e^{-i \theta}$ to $\partial \Omega_{R}\left(x_{0}\right)$ as above, and define $d_{1}$ to be the degree on $\partial \Omega_{R}\left(x_{0}\right)$ of this extension. We represent $p$ in $A_{r, R}\left(x_{0}\right)$ by $p=\zeta(\rho, \theta) e^{i\left(\theta+2 d_{1} \theta+\phi(\rho, \theta)\right)}$.

Case 2: $x_{0} \in \mathcal{V}$. Without loss of generality, we again assume $x_{0}=(0,0)$, so that $\Gamma_{r, R}^{+}$is along $\Gamma_{H}^{B}$ and $\Gamma_{r, R}^{-}$is along $\Gamma_{V}^{L}$, the left portion of the vertical boundary. Note that the angle measured for every point on $\Gamma_{r, R}^{-}$with respect to the positively oriented horizontal line at $x_{0}$ is $\pi / 2$. Also, recall that $p \cdot \tau=p_{1}$ on $\Gamma_{H}^{B}$ and $p=(-1,0)$ on $\Gamma_{V}^{L}$.

If $p_{1}$ changes sign on $\Gamma_{r, R}^{ \pm}$, we consider $p e^{-2 i \theta}$. Then, the first component of $p e^{-2 i \theta}$ does not change sign on $\Gamma_{r, R}^{ \pm}$, and we can extend $p e^{-2 i \theta}$ to a $\tilde{p}$ in $\partial \Omega_{R}\left(x_{0}\right)$ so that $|\tilde{p}| \geq 1 / 2$ on $B_{R}\left(x_{0}\right) \cap \Gamma$ and $|\tilde{p} \cdot \nu| \leq 1 / 4$ in $B_{R}\left(x_{0}\right) \cap \Gamma_{H}$. We denote by $d_{1}$ the degree on $\partial \Omega_{R}\left(x_{0}\right)$ of $\tilde{p}$, and consider the following representation of $p$ in polar coordinates $p=\zeta(\rho, \theta) e^{i\left(2 \theta+4 d_{1} \theta+\phi(\rho, \theta)\right)}$, with $\phi$ smooth single valued function in $A_{r, R}\left(x_{0}\right)$.

If $p_{1}$ does not change its sign on $\Gamma_{r, R}^{ \pm}$, we extend $p$ on $\partial \Omega_{R}\left(x_{0}\right)$, define $\tilde{d}$ to be the degree of this extension, and represent $p$ in polar coordinates as $p=\zeta(\rho, \theta) e^{i(4 \tilde{d} \theta+\phi(\rho, \theta))}$.

Case 3: $x_{0} \in \Gamma_{V}$, and $B_{R}\left(x_{0}\right) \cap \Gamma_{H}=\emptyset$. By the assumptions on $R$, we have that $p \cdot \nu=$ constant $\neq 0$ on $B_{R}\left(x_{0}\right) \cap \Gamma_{V}$, hence we just consider $d=\operatorname{deg}\left(\frac{p}{|p|}, \partial \Omega_{R}\left(x_{0}\right)\right)$, and the representation: $p=\zeta(\rho, \theta) e^{i(2 d \theta+\phi(\rho, \theta))}$.

Let $\gamma$ be as in Proposition 3.3, using the representations just introduced, we are able to provide energy lower bounds analogous to the ones of [32].

Lemma 3.4. Let $x_{0} \in \bar{\Omega}$, and $\varepsilon \leq r<R<1 / 4$. Suppose that $\frac{1}{2} \leq|p| \leq 1$ on $A_{r, R}\left(x_{0}\right) ;|p \cdot \nu| \leq \frac{1}{4}$ on $\Gamma_{r, R}^{ \pm} \cap \Gamma_{H}$, and there are constants $C_{1}, C_{2}$ with $E_{\varepsilon}(p) \leq C_{1}|\ln \varepsilon|$, and $\int_{\Omega_{\varepsilon} \gamma\left(x_{0}\right)}\left(\frac{1}{2 \varepsilon^{2}}\left(1-|p|^{2}\right)^{2}+\sigma|\mathbf{e}-p|^{2}\right) d x+\frac{1}{\varepsilon} \int_{\Gamma_{\varepsilon}^{H}\left(x_{0}\right)}(p \cdot \nu)^{2} d s \leq C_{2}$.

Then, there exists a constant $C$ such that

(a) If $B_{R}\left(x_{0}\right) \subset \Omega, \operatorname{deg}\left(p, \partial B_{R}\left(x_{0}\right)\right) \neq 0$, then $\frac{1}{2} \int_{A_{r, R}\left(x_{0}\right)}|\nabla p|^{2} d x \geq \pi \ln \frac{R}{r}+C$.

(b) If $x_{0} \in \Gamma_{H}, B_{R}\left(x_{0}\right) \cap \Gamma_{V}=\emptyset$, and $p \cdot \tau$ does not change sign in $\Gamma_{r, R}^{ \pm}$, then whenever $\tilde{d} \neq 0$, for $\tilde{d}$ defined as in Case 1 above, we have

$$
\frac{1}{2} \int_{A_{r, R}\left(x_{0}\right)}|\nabla p|^{2} d x \geq \frac{(2 \tilde{d})^{2} \pi}{2} \ln \frac{R}{r}+C \geq 2 \pi \ln \frac{R}{r}+C .
$$

(c) If $x_{0} \in \Gamma_{H}, B_{R}\left(x_{0}\right) \cap \Gamma_{V}=\emptyset$, and $p \cdot \tau$ changes sign in $\Gamma_{r, R}^{ \pm}$, then for $d_{1}$ defined as in Case 1 above, we have

$$
\frac{1}{2} \int_{A_{r, R}\left(x_{0}\right)}|\nabla p|^{2} d x \geq \frac{\left(1+2 d_{1}\right)^{2} \pi}{2} \ln \frac{R}{r}+C \geq \frac{\pi}{2} \ln \frac{R}{r}+C .
$$

(d) If $x_{0} \in \mathcal{V}$, and $p_{1}$ has same sign on $\Gamma_{r, R}^{ \pm}$, then whenever $\tilde{d} \neq 0$, for $\tilde{d}$ defined as in Case 2 above, we have

$$
\frac{1}{2} \int_{A_{r, R}\left(x_{0}\right)}|\nabla p|^{2} d x \geq(4 \tilde{d})^{2} \frac{\pi}{4} \ln \frac{R}{r}+C \geq 4 \pi \ln \frac{R}{r}+C .
$$

(e) If $x_{0} \in \mathcal{V}$, and $p_{1}$ has opposite sign on $\Gamma_{r, R}^{ \pm}$, then for $d_{1}$ defined as in Case 2 above, we have

$$
\frac{1}{2} \int_{A_{r, R}\left(x_{0}\right)}|\nabla p|^{2} d x \geq\left(2+4 d_{1}\right)^{2} \frac{\pi}{4} \ln \frac{R}{r}+C \geq \pi \ln \frac{R}{r}+C .
$$


(f) If $x_{0} \in \Gamma_{V}$, and $B_{R}\left(x_{0}\right) \cap \Gamma_{H}=\emptyset$, then whenever $d \neq 0$, for $d$ defined as in Case 3 above, we have

$$
\frac{1}{2} \int_{A_{r, R}\left(x_{0}\right)}|\nabla p|^{2} d x \geq \frac{(2 d)^{2} \pi}{2} \ln \frac{R}{r}+C \geq 2 \pi \ln \frac{R}{r}+C .
$$

Proof. The energy bounds follow from the arguments of [32] and [23], with modifications analogous to the ones presented in Proposition 3.5 below.

To narrow down the possible locations of near boundary defects, we need to account for annuli with center not on a corner, whose outer circles intersect both the horizontal and vertical boundaries. The cases in which the center is on the horizontal side (see Figure 2) is discussed in Proposition 3.5. If the center is on the vertical side, due to the boundary conditions, under the assumptions made, only $B_{r}\left(x_{0}\right) \cap \Gamma_{V}=\emptyset$ is possible, this case is addressed in Proposition 3.6.
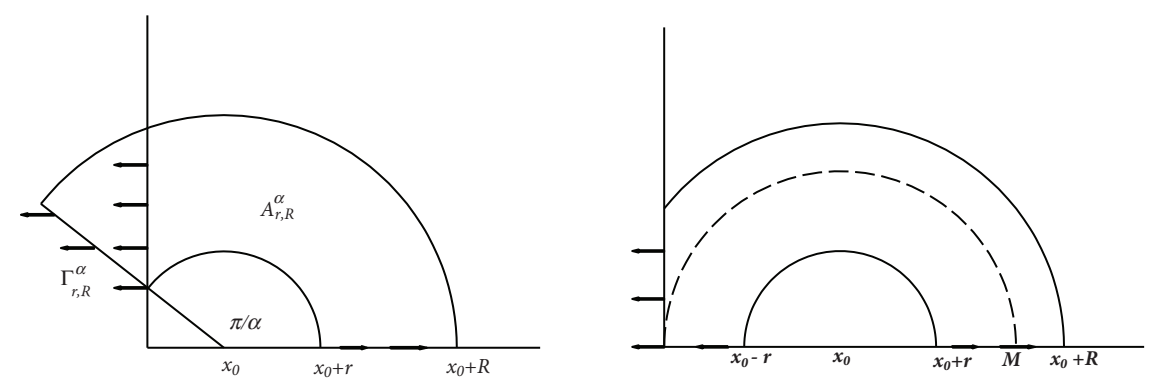

FIG. 2. The left figure depicts the annular sector $A_{r, R}^{\alpha}$, and the constant extension of $p$ outside $\Omega$. The right figure depicts the annular domains $A_{r, M}$ and $A_{M, R}$, and $p$ on their horizontal and vertical boundaries.

Proposition 3.5. Let $x_{0} \in \Gamma_{H}$, and $\varepsilon \leq r<R \leq 1 / 2$. Assume $B_{R}\left(x_{0}\right) \cap \Gamma_{V} \neq \emptyset$, and $p_{1}$ changes its sign on $\Gamma_{r, R}^{ \pm}$. Suppose that $\frac{1}{2} \leq|p| \leq 1$ on the annulus $A_{r, R}\left(x_{0}\right)$, $|p \cdot \nu| \leq \frac{1}{4}$ on $\Gamma_{r, R}^{ \pm} \cap \Gamma_{H}$, and there are constants $C_{1}, C_{2}$ with $E_{\varepsilon}(p) \leq C_{1}|\ln \varepsilon|$ and

$$
\int_{\Omega_{\varepsilon \gamma}\left(x_{0}\right)}\left(\frac{1}{2 \varepsilon^{2}}\left(1-|p|^{2}\right)^{2}+\sigma|\mathbf{e}-p|^{2}\right) d x+\frac{1}{\varepsilon} \int_{\Gamma_{\varepsilon \gamma}^{H}\left(x_{0}\right)}(p \cdot \nu)^{2} d s \leq C_{2} .
$$

There exists a constant $C$ such that if $B_{r}\left(x_{0}\right) \cap \Gamma_{V} \neq \emptyset$, then

$$
\frac{1}{2} \int_{A_{r, R}\left(x_{0}\right)}|\nabla p|^{2} d x \geq \frac{\alpha \pi\left(1+2 d_{\alpha}\right)^{2}}{2} \ln \frac{R}{r}+C \geq \frac{\alpha \pi}{2} \ln \frac{R}{r}+C
$$

for some $1<\alpha<2$; and

$$
\frac{1}{2} \int_{A_{r, R}\left(x_{0}\right)}|\nabla p|^{2} d x \geq \frac{\pi}{2} \ln \frac{R}{r}+C \quad \text { if } B_{r}\left(x_{0}\right) \cap \Gamma_{V}=\emptyset .
$$

Proof. If $\partial B_{r}\left(x_{0}\right) \cap \Gamma_{V} \neq \emptyset$, without loss of generality, we may assume $\Gamma_{r, R}^{-} \subset \Gamma_{V}^{L}$, and $\Gamma_{r, R}^{+}$along $\Gamma_{H}^{B}$. Let $\pi / \alpha$ be the angle for the point $\partial B_{r}\left(x_{0}\right) \cap \Gamma_{V}$, measured with respect to the positively oriented line at $x_{0} \in \Gamma_{H}^{B}$. See Figure 2 for the case 
$B_{r}\left(x_{0}\right) \cap \Gamma_{V} \neq \emptyset, r>\operatorname{dist}\left(x_{0}, \mathcal{V}\right)$. Note that $1<\alpha<2$, so that $\frac{\pi}{2}<\frac{\pi}{\alpha}<\pi$. Consider the annular sector with central angle $\pi / \alpha$, and denote it by $A_{r, R}^{\alpha}$. This sector is bordered by two straight lines through $x_{0}$ : one is $\Gamma_{r, R}^{+}$, and the other is denoted by $\Gamma_{r, R}^{\alpha}$. Since $p=\nu$ on $\Gamma_{V}^{L}$, we may extend $p$ to be a constant vector field on $A_{r, R}^{\alpha} \backslash A_{r, R}$, so that this extension, still denoted by $p$, satisfies $\int_{A_{r, R}}|\nabla p|^{2} \geq \int_{A_{r, R}^{\alpha}}|\nabla p|^{2}-C$.

If $p_{1}$ changes its sign, since this means that we have $p_{1}>0$ on $\Gamma_{r, R}^{+}$, and $p_{1}=-1$ on $\Gamma_{r, R}^{\alpha}$, we may estimate the cost of the vortex on $A_{r, R}^{\alpha}$, in the same way as done for the case of the half or a quarter annular domains considered in Lemma 3.4. Noticing that in this situation the first component of $p e^{-i \alpha \theta}$ does not change its sign on $\Gamma_{r, R}^{+}$and $\Gamma_{r, R}^{\alpha}$, we define $d_{\alpha}$, the degree of the extension of $p e^{-i \alpha \theta}$ on $\partial \Omega_{R}\left(x_{0}\right)$. And, represent $p$ in polar coordinates $(\rho, \theta)$, centered at $x_{0}$, with $\rho=\left|x-x_{0}\right|$, and $\theta$ measured with respect to the positively oriented line at $x_{0} \in \Gamma_{H}^{B}$, that is $p=\zeta(\rho, \theta) e^{i\left(\alpha \theta+2 \alpha d_{\alpha} \theta+\phi(\rho, \theta)\right)}$, with $\phi$ smooth single-valued function in $A_{r, R}^{\alpha}$. Furthermore, we may assume that

$$
|\phi| \leq C|p \cdot \nu| \quad \text { on } \Gamma_{r, R}^{+} \quad \text { and } \quad \phi=0 \quad \text { on } \Gamma_{r, R}^{\alpha} .
$$

In fact, from $|p \cdot \nu|=|\zeta||\sin \phi| \geq \frac{1}{2}|\sin \phi|$ at $\theta=0$, we have $|\phi| \leq C|p \cdot \nu|$ on $\Gamma_{r, R}^{+}$. While, from $p=-\mathbf{e}_{1}$ on $\Gamma_{r, R}^{\alpha}$ we may choose $\phi=0$ there. Set $k=\alpha\left(1+2 d_{\alpha}\right)$, then

$$
\begin{aligned}
|\nabla p|^{2} & =|\nabla \zeta|^{2}+\zeta^{2}\left\{\left|\frac{\partial \phi}{\partial \rho}\right|^{2}+\frac{1}{\rho^{2}}\left(k+\frac{\partial \phi}{\partial \theta}\right)^{2}\right\} \geq \zeta^{2}\left\{|\nabla \phi|^{2}+\frac{1}{\rho^{2}}\left(k^{2}+2 k \frac{\partial \phi}{\partial \theta}\right)\right\} \\
& =\frac{k^{2}}{\rho^{2}}+k^{2} \frac{\zeta^{2}-1}{\rho^{2}}+\frac{2 k}{\rho^{2}} \frac{\partial \phi}{\partial \theta}+2 k \frac{\zeta^{2}-1}{\rho^{2}} \frac{\partial \phi}{\partial \theta}+\zeta^{2}|\nabla \phi|^{2} \\
& :=I_{1}+I_{2}+I_{3}+I_{4}+I_{5} .
\end{aligned}
$$

The first term is the dominant term that gives the desired lower bound, since

$$
\int_{A_{r, R}^{\alpha}} I_{1}=k^{2} \int_{0}^{\frac{\pi}{\alpha}} \int_{r}^{R} \frac{1}{\rho} d \rho d \theta=k^{2} \frac{\pi}{\alpha} \ln \frac{R}{r}=\pi \alpha\left(1+2 d_{\alpha}\right)^{2} \ln \frac{R}{r} .
$$

To estimate $I_{2}, I_{3}$ and $I_{4}$, we split the domain into $A_{r, R}^{\alpha}=A_{r, \varepsilon^{\gamma}}^{\alpha} \cup A_{\varepsilon^{\gamma}, R}^{\alpha}$, define $B_{R}^{\alpha}$ to be the sector with radius $R$ and central angle $\alpha$, and proceed as follows.

In $I_{2}$, since $1-\zeta^{2}=0$ in $A_{r, R}^{\alpha} \backslash A_{r, R}$, we use (3.15) in $A_{r, R}$, to gather

$$
\begin{gathered}
\left|\int_{A_{r, \varepsilon \gamma}} \frac{1-\zeta^{2}}{\rho^{2}}\right| \leq \\
\left.\leq \varepsilon^{2} \int_{B_{\varepsilon} \gamma} \frac{\left(1-\zeta^{2}\right)^{2}}{\varepsilon^{2}}\right]^{\frac{1}{2}}\left[\int_{0}^{\pi / \alpha} \int_{r}^{\varepsilon^{\gamma}} \frac{1}{\rho^{3}} d \rho d \theta\right]^{\frac{1}{2}} \\
\leq\left[C_{2} \varepsilon^{2}\left(\frac{1}{r^{2}}-\frac{1}{\varepsilon^{2 \gamma}}\right)\right]^{\frac{1}{2}} \leq C
\end{gathered}
$$

and the upper bound estimate from Lemma 3.1, to derive

$$
\begin{aligned}
\left|\int_{A_{\varepsilon} \gamma, R} \frac{1-\zeta^{2}}{\rho^{2}}\right| & \leq\left[\varepsilon^{2} \int_{B_{R}} \frac{\left(1-\zeta^{2}\right)^{2}}{\varepsilon^{2}}\right]^{\frac{1}{2}}\left[\int_{0}^{\pi / \alpha} \int_{\varepsilon^{\gamma}}^{R} \frac{1}{\rho^{3}} d \rho d \theta\right]^{\frac{1}{2}} \\
& \leq\left[C_{1} \varepsilon^{2}|\ln \varepsilon|\left(\frac{1}{\varepsilon^{2 \gamma}}-\frac{1}{R^{2}}\right)\right]^{\frac{1}{2}} \leq o(1) .
\end{aligned}
$$

being $3 / 4<\gamma<1$. We thus conclude $\left|I_{2}\right| \leq C+o(1)$. 
To estimate $I_{3}$, we use (3.16) to obtain that $\int_{A_{r, R}^{\alpha}} \frac{1}{\rho^{2}} \frac{\partial \phi}{\partial \theta}=\int_{r}^{R} \int_{0}^{\pi / \alpha} \frac{\partial \phi}{\partial \theta} d \theta \frac{d \rho}{\rho}=$ $\int_{r}^{R}\left(\phi\left(\rho, \frac{\pi}{\alpha}\right)-\phi(\rho, 0)\right) \frac{d \rho}{\rho} \leq C \int_{\Gamma_{r, R}^{+}}|p \cdot \nu| \frac{d \rho}{\rho}$. And, again apply (3.15) and Lemma 3.1, to see that as $\varepsilon \rightarrow 0$, it holds $\int_{\Gamma_{r, \varepsilon}^{+} \gamma}|p \cdot \nu| \frac{d \rho}{\rho} \leq\left[\int_{\Gamma_{r, \varepsilon}^{+} \gamma}|p \cdot \nu|^{2} d \rho \int_{\Gamma_{r, \varepsilon}^{+}} \frac{1}{\rho^{2}} d \rho\right]^{\frac{1}{2}} \leq$ $C_{2}\left[\varepsilon\left(\frac{1}{r}-\frac{1}{\varepsilon^{\gamma}}\right)\right]^{\frac{1}{2}} \leq C$, and, similarly $\int_{\Gamma_{\varepsilon \gamma, R}^{+}}|p \cdot \nu| \frac{1}{\rho} d \rho \leq\left[C_{1} \varepsilon|\ln \varepsilon|\left(\frac{1}{\varepsilon^{\gamma}}-\frac{1}{R}\right)\right]^{\frac{1}{2}} \leq o(1)$.

For $I_{4}$, we have that $\int_{A_{\varepsilon \gamma, R}^{\alpha}} \frac{1}{\rho}\left(1-\zeta^{2}\right) \frac{1}{\rho} \frac{\partial \phi}{\partial \theta} \leq \frac{1}{\varepsilon^{\gamma}}\left[\int_{B_{R}^{\alpha}}\left(1-\zeta^{2}\right)^{2} \int_{A_{r, R}^{\alpha}}|\nabla \phi|^{2}\right]^{\frac{1}{2}} \leq$ $\frac{1}{\varepsilon^{\gamma}}\left[C_{1} \varepsilon^{2}|\ln \varepsilon|\|\nabla \phi\|_{2}^{2}\right]^{\frac{1}{2}}=o(1)\|\nabla \phi\|_{L^{2}\left(A_{r, R}^{\alpha}\right)} \leq C+o(1)\|\nabla \phi\|_{L^{2}\left(A_{r, R}^{\alpha}\right)}^{2} ;$ and $\int_{A_{r, \varepsilon}^{\alpha} \gamma} \frac{1}{\rho}(1-$ $\left.\zeta^{2}\right) \frac{1}{\rho} \frac{\partial \phi}{\partial \theta} \leq C\|\nabla \phi\|_{2} \leq C+\frac{1}{8}\|\nabla \phi\|_{L^{2}\left(A_{r, R}^{\alpha}\right)}^{2}$. Finally, we notice that for $I_{5}$, it holds $\int_{A_{r, R}^{\alpha}} \zeta^{2}|\nabla \phi|^{2} \geq \frac{1}{4}\|\nabla \phi\|_{L^{2}\left(A_{r, R}^{\alpha}\right)}^{2}$, which can be used to control $I_{4}$. Putting all the terms together, we find: $\int_{A_{r, R}}|\nabla p|^{2} \geq \pi \alpha\left(1+2 d_{\alpha}\right)^{2} \ln \frac{R}{r}-C$, for a constant $C$ depending only on $\Omega, C_{1}, C_{2}$, and $\gamma$.

Next, we consider the case $B_{r}\left(x_{0}\right) \cap \Gamma_{V}=\emptyset$. Without loss of generality, we assume $\Gamma_{r, R}^{-} \cap \Gamma_{V}^{L} \neq \emptyset$, and $\Gamma_{r, R}^{+}$along $\Gamma_{H}^{B}$. We split the domain $A_{r, R}=A_{r, M} \cup A_{M, R}$, where $M$ is the distance from $x_{0}$ to the left bottom corner, so that $A_{r, M}$ becomes a half annulus, and $A_{M, R}^{-} \subset \Gamma_{V}^{L}$. Note that, $M=r$ is an allowed possibility. We apply Lemma 3.4 part (c), and follow the proof above, assuming now $\alpha=1$, we conclude $\int_{A_{r, R}}|\nabla p|^{2} \geq \int_{A_{r, M}}|\nabla p|^{2}+\int_{A_{M, R}}|\nabla p|^{2} \geq \pi \ln \frac{M}{r}+\pi \ln \frac{R}{M}+C \geq \pi \ln \frac{R}{r}+C . \mathrm{c}$

Proposition 3.6. Let $x_{0} \in \Gamma_{V}$, and $\varepsilon \leq r<R \leq 1 / 2$. Assume $B_{R}\left(x_{0}\right) \cap \Gamma_{H} \neq \emptyset$, and $p_{1}$ changes its sign on $\Gamma_{r, R}^{ \pm}$. Suppose that $\frac{1}{2} \leq|p| \leq 1$ on the annulus $A_{r, R}\left(x_{0}\right)$, $|p \cdot \nu| \leq \frac{1}{4}$ on $\Gamma_{r, R}^{ \pm} \cap \Gamma_{H}$, and there are constants $C_{1}, C_{2}$ with $E_{\varepsilon}(p) \leq C_{1}|\ln \varepsilon|$ and

$$
\int_{\Omega_{\varepsilon}\left(x_{0}\right)}\left(\frac{1}{2 \varepsilon^{2}}\left(1-|p|^{2}\right)^{2}+\sigma|\mathbf{e}-p|^{2}\right) d x+\frac{1}{\varepsilon} \int_{\Gamma_{\varepsilon \gamma}^{H}\left(x_{0}\right)}(p \cdot \nu)^{2} d s \leq C_{2} .
$$

Then, there exists a constants $C$ such that we have, for some $1<\alpha<2$,

$$
\frac{1}{2} \int_{A_{r, R}\left(x_{0}\right)}|\nabla p|^{2} d x \geq \frac{\alpha \pi\left(1+d_{\alpha}\right)^{2}}{2} \ln \frac{R}{r}+C \geq \frac{\alpha \pi}{2} \ln \frac{R}{r}+C \text { if } B_{r}\left(x_{0}\right) \cap \Gamma_{H} \neq \emptyset .
$$

Proof. As in the proof of Proposition 3.5, we assume $\Gamma_{r, R}^{-} \subset \Gamma_{V}^{L}$ and $\Gamma_{r, R}^{+} \cap \Gamma_{H}^{B} \neq \emptyset$. In fact, our assumptions that $p_{1}$ changes its sign on $\Gamma_{r, R}^{ \pm}, \frac{1}{2} \leq|p| \leq 1$ on $A_{r, R}\left(x_{0}\right)$, and $|p \cdot \nu| \leq \frac{1}{4}$ on $\Gamma_{r, R}^{ \pm} \cap \Gamma_{H}$, together with the boundary conditions, imply that $\Gamma_{r, R}^{+} \subset \Gamma_{H}^{B}$. We extend the ray between the center $x_{0}$, and the point $\partial B_{r}\left(x_{0}\right) \cap \Gamma_{H}^{B}$, so to meet $\partial B_{s}\left(x_{0}\right)$, for any $r<s \leq R$, and denote this extended ray by $\Gamma_{r, s}^{\alpha}$. We then let $A_{r, s}^{\alpha}$ be the annular sector bordered by $\Gamma_{r, s}^{-}$and $\Gamma_{r, s}^{\alpha}$, and $\pi / \alpha$ its central angle, note that $\alpha$ does not depend on $r<s \leq R$. Our assumptions imply that $p_{1}>\sqrt{3} / 4$ and $\left|p_{2}\right|<1 / 4$ on $\Gamma_{r, R}^{+}$, and

$$
\int_{\Gamma_{r, R}^{+}} p_{2}^{2} \leq C_{1} \varepsilon|\ln \varepsilon| \quad \text { and } \quad \int_{\Gamma_{r, \varepsilon}^{+} \gamma} p_{2}^{2} \leq C_{2} \varepsilon
$$

Next, we assign continuous boundary values holding these conditions also on the other parts of the boundary of $A_{r, R}^{\alpha} \backslash A_{r, R}$, with (3.18) valid on $\Gamma_{r, R}^{\alpha}$ and $\Gamma_{r, \varepsilon^{\gamma}}^{\alpha}$. And, we consider the $\mathbb{S}^{1}$-valued harmonic extension, of these boundary values, to $A_{r, R}^{\alpha} \backslash A_{r, R}$. This allows us to define an extension $\tilde{p}$ of $p$ in $A_{r, R}^{\alpha}$, for which $\tilde{p_{1}}$ has 
opposite sign in $\Gamma_{r, R}^{-}$and $\Gamma_{r, R}^{\alpha}$, and $\int_{A_{r, R}}|\nabla p|^{2} \geq \int_{A_{r, R}^{\alpha}}|\nabla \tilde{p}|^{2}-C$. Considering polar coordinates centered at $x_{0}$, so that $A_{r, R}^{\alpha}=\{(\rho, \theta): r \leq \rho \leq R,-\beta \leq \theta \leq \pi / 2\}$ where $\beta=\pi\left(\frac{1}{\alpha}-\frac{1}{2}\right)$, we can proceed as in Proposition 3.5. Indeed, the first component of $p e^{-i(\alpha(\theta+\beta))}$ has now constant sign on $\Gamma_{r, R}^{-}$and $\Gamma_{r, R}^{\alpha}$, therefore we may write $p(\rho, \theta)=$ $\zeta(\rho, \theta) e^{i(\alpha(1+2 d)(\theta+\beta)+\phi(\rho, \theta))}$, with $\phi$ single valued, and follow our previous proof.

REMARK 3.1. If in Propositions 3.5 and 3.6, $p_{1}$ does not change sign, we can extend $p$ as done in Lemma 3.4, and if the degree $d$ of this extension is non-zero, the arguments of our previous proofs imply $\frac{1}{2} \int_{A_{r, R}\left(x_{0}\right)}|\nabla p|^{2} d x>\pi \ln \frac{R}{r}+C$.

4. Convergence. Using the estimates of Section 3.2, we can show that the number of near defects, and the size of the sets containing them are uniformly bounded in $\varepsilon$ small. From this, thanks to the results of Section 3.3, we obtain a lower bound for the energy, which, combined with the upper bound of Section 3.1, implies that for small $\varepsilon$ only two boundary near defects are allowed in global minimizers.

For a minimizer $p^{\varepsilon}$ of $E_{\varepsilon}$, we define the set

$$
S_{\varepsilon}=\left\{x \in \bar{\Omega}:\left|p^{\varepsilon}(x)\right|<\frac{1}{2}\right\} \cup\left\{x \in \Gamma_{H}:\left|p^{\varepsilon} \cdot \nu\right|>\frac{1}{4}\right\}
$$

LEMmA 4.1. There exist $N_{0}=N_{0}(\Omega), K>1$, and points $a_{1}^{\varepsilon}, a_{2}^{\varepsilon}, \ldots, a_{I_{\varepsilon}}^{\varepsilon} \in S_{\varepsilon}$ such that $I_{\varepsilon} \leq N_{0}$, and $S_{\varepsilon} \subset\left(\bigcup_{i=1}^{I_{\varepsilon}} \Omega_{K \varepsilon}\left(a_{i}^{\varepsilon}\right)\right)$, with $\left\{\Omega_{K \varepsilon}\left(a_{i}^{\varepsilon}\right)\right\}_{1 \leq i \leq I_{\varepsilon}}$ mutually disjoint sets, and $\left|a_{i}^{\varepsilon}-a_{j}^{\varepsilon}\right|>8 K \varepsilon$, whenever $i \neq j$.

Proof. The lemma can be proven as the analogous results in [32] and [1,2]. In fact, Lemma 3.2 and Proposition 3.3 allow us to follow the line of proof of Proposition 5.1 in [1]. The bound $\left|a_{i}^{\varepsilon}-a_{j}^{\varepsilon}\right|>8 K \varepsilon$ is obtained as in Lemma 4.4 of [2].

REMARK 4.1. Let $\varepsilon<\varepsilon_{0}$, with $\varepsilon_{0}$ defined as in Proposition 3.1. If we take $\varepsilon \rightarrow 0$, Lemma 4.1 implies that there are an integer $I$, elements $c_{k} \in \bar{\Omega}, k=1 \ldots N \leq I$, and a subsequence $\left\{\varepsilon_{n}\right\}$ such that it holds $I_{\varepsilon_{n}}=I$, and $a_{i}^{\varepsilon_{n}} \rightarrow c_{k}$ for some $k$ depending on $i$. Note that distinct sequences $a_{i}^{\varepsilon_{n}}, a_{j}^{\varepsilon_{n}}$ could converge to the same $c_{k}$, hence $N \leq I$.

We set $s_{1}=\min \left\{\left|c_{i}-c_{j}\right|: c_{i} \neq c_{j}\right\}, s_{2}=\min \left\{\operatorname{dist}\left(c_{j} ; \mathcal{V}\right): c_{i} \notin \mathcal{V}\right\}$, and $s_{3}=$ $\min \left\{\operatorname{dist}\left(c_{j} ; \Gamma\right): c_{i} \notin \Gamma\right\} ;$ and define $s_{0}$ as $s_{0}:=\frac{1}{4} \min \left\{s_{1}, s_{2}, s_{3}, 1\right\}$. Additionally, passing to another subsequence if necessary, still denoted by $\left\{\varepsilon_{n}\right\}$, we can assume $K \varepsilon_{n}<s_{0} / 4$, and $\left|a_{i}^{\varepsilon_{n}}-c_{k}\right|<s_{0} / 4$, if $a_{i}^{\varepsilon_{n}} \rightarrow c_{k}$.

We also divide the $c_{k}$ 's in three subsets: $\omega_{k} \equiv c_{k} \in \Omega, k=1 . . N_{\omega} ; b_{k} \equiv c_{k} \in$ $\Gamma_{H} \cup \Gamma_{V}, k=1 . . N_{b} ; v_{k} \equiv c_{k} \in \mathcal{V}, k=1 . . N_{v}$, where $N_{\omega}+N_{b}+N_{v}=N$.

For $s>0$, we introduce the set $\Omega_{s}:=\Omega \backslash\left(\bigcup_{i=1}^{N} \Omega_{s}\left(c_{i}\right)\right)$.

LEMMA 4.2. Given a sequence of global minimizers $\left\{p_{\varepsilon}\right\}:$ for $\left\{\varepsilon_{n}\right\}$ and $s_{0}$ as in Remark 4.1, we have that for any fixed $0<s<s_{0}$, as $\varepsilon_{n}$ converges to zero, there exist constants $C_{1}=C(\Omega, \sigma)$ and $C_{2}=C(\Omega, \sigma)$ such that

$$
E_{\varepsilon_{n}}\left(p_{\varepsilon_{n}} ; \bigcup_{i=1}^{N} \Omega_{s}\left(c_{i}\right)\right) \geq \pi\left(N_{\omega}+\frac{1}{2} N_{b}+\frac{1}{2} N_{v}\right) \ln \frac{s}{\varepsilon_{n}}+C_{1},
$$

and $\quad E_{\varepsilon_{n}}\left(p_{\varepsilon_{n}} ; \Omega_{s}\right) \leq \pi\left(N_{\omega}+\frac{1}{2} N_{b}+\frac{1}{2} N_{v}\right)|\ln s|+C_{2}$.

Moreover, $N_{\omega}=N_{v}=0$, and $N_{b}=2$, that is there are exactly two near defects $b_{1}^{*}, b_{2}^{*} \in \Gamma_{H}$, and, they are on opposite sides of the horizontal boundary.

Proof. As $\varepsilon_{n}$ tends to zero, we assume $K \varepsilon_{n}<s / 4$, and $\left|a_{i}^{\varepsilon_{n}}-c_{k}\right|<s / 4$ if $a_{i}^{\varepsilon_{n}} \rightarrow c_{k}$. The ball construction of [31], and the modification dealing with boundary 
vortices presented in Lemma 7.1 in [2], can be directly applied to our problem, and we refer the reader to [2] for details. In the following, we just emphasize a few issues.

If $a_{i}^{\varepsilon_{n}} \in \Omega$, we pick a small enough ball centered at $a_{i}^{\varepsilon_{n}}$, and define the degree of $p_{\varepsilon_{n}}$ in the standard way. If $a_{i}^{\varepsilon_{n}} \in \Gamma$, since the sets $\Omega_{K \varepsilon_{n}}\left(a_{i}^{\varepsilon_{n}}\right)$ of Lemma 4.1 are mutually disjoint, with $\left|a_{i}^{\varepsilon}-a_{j}^{\varepsilon}\right|>8 K \varepsilon$ whenever $i \neq j$, we consider the annulus $A_{K \varepsilon, 2 K \varepsilon}\left(a_{i}^{\varepsilon}\right)$, and associate to $a_{i}^{\varepsilon}$ the degree of the extension $\tilde{p}_{\varepsilon_{n}}$ defined in Section 3.3.

Under our assumptions, the sets $\Omega_{K \varepsilon_{n}}\left(a_{i}^{\varepsilon_{n}}\right)$ of Lemma 4.1, which have centers tending to some $\omega_{k}$, do not intersect $\Gamma$, that is $\Omega_{K \varepsilon_{n}}\left(a_{i}^{\varepsilon_{n}}\right)=B_{K \varepsilon_{n}}\left(a_{i}^{\varepsilon_{n}}\right)$, and their union can be included in a set $\widetilde{\Omega} \Subset \Omega$.

In the spirit of Remark 7.3 in [2], we start the process by including only the sets $\Omega_{K \varepsilon_{n}}\left(a_{i}^{\varepsilon_{n}}\right) \subset \widetilde{\Omega}$, for which $p_{\varepsilon_{n}}$ has non-zero degree, the sets $\Omega_{K \varepsilon_{n}}\left(a_{i}^{\varepsilon_{n}}\right)$ whose center tends to a $b_{k} \in \Gamma$ and $p_{\varepsilon_{n}} \cdot \tau$ changes sign on $\Gamma_{K \varepsilon, 2 K \varepsilon}^{ \pm}$, and the $\Omega_{K \varepsilon_{n}}\left(a_{i}^{\varepsilon_{n}}\right)$ 's whose center tends to a $v_{k} \in \Gamma$ and $\left(p_{\varepsilon_{n}}\right)_{1}$ changes sign on $\Gamma_{K \varepsilon, 2 K \varepsilon}^{ \pm}$.

For the balls in $\widetilde{\Omega}$ the classical construction method of [31] can be used. Instead, in the merging process for the sets $\Omega_{K \varepsilon_{n}}\left(a_{i}^{\varepsilon_{n}}\right)$ with centers tending to $b_{k}$ 's or $v_{k}$ 's, we can apply the strategy of Lemma 7.1 in [2]. Because of our assumptions these sets are either balls contained in $\Omega$ or boundary balls with center on $\Gamma$, the merging of an interior ball touching the boundary or of an interior and a boundary ball touching each other is handled by creating a new boundary ball with the center on $\Gamma$. Thus, in all situations the results of Section 3.3 are sufficient to derive the energy lower bounds during the merging process, and the ball construction in Lemma 7.1 of [2] leads to $E_{\varepsilon_{n}}\left(p_{\varepsilon_{n}} ; \bigcup_{i=1}^{N} \Omega_{s}\left(c_{i}\right)\right) \geq \pi\left(N_{\omega}+\frac{1}{2} N_{b}+\frac{1}{2} N_{v}\right) \ln \frac{s}{\varepsilon_{n}}+C_{1}$. But, from Proposition 3.1, we know that $E_{\varepsilon_{n}}\left(p_{\varepsilon_{n}} ; \Omega\right) \leq \pi\left|\ln \varepsilon_{n}\right|+C(1+\sigma)$. Hence, we have $0 \leq E_{\varepsilon_{n}}\left(p_{\varepsilon_{n}} ; \Omega_{s}\right) \leq \pi\left|\ln \varepsilon_{n}\right|-\pi\left(N_{\omega}+\frac{1}{2} N_{b}+\frac{1}{2} N_{v}\right) \ln \frac{s}{\varepsilon_{n}}+C_{2}$, which gives $0 \leq$ $E_{\varepsilon_{n}}\left(p_{\varepsilon_{n}} ; \Omega_{s}\right) \leq \pi\left(N_{\omega}+\frac{1}{2} N_{b}+\frac{1}{2} N_{v}\right)|\ln s|+\pi\left(1-N_{\omega}-\frac{1}{2} N_{b}-\frac{1}{2} N_{v}\right)\left|\ln \varepsilon_{n}\right|+C_{2}$. This, for $s$ fixed, and $\varepsilon_{n}$ going to zero, implies $N_{\omega}+\frac{1}{2} N_{b}+\frac{1}{2} N_{v} \leq 1$, as otherwise the right hand side would be eventually negative. Now, $p_{\varepsilon_{n}} \cdot \tau$ must change sign at least once on each component of $\Gamma_{H}$, due to the Dirichlet boundary condition on $\Gamma_{V}$, so we also have $N_{\omega}+\frac{1}{2} N_{b}+\frac{1}{2} N_{v} \geq 1$, and we gather $N_{\omega}+\frac{1}{2} N_{b}+\frac{1}{2} N_{v}=1$. But, again the boundary condition does not allow for $N_{\omega}=1, N_{b}=N_{v}=0$, and requires at least one sign change on each component of $\Gamma_{H}$, that is $N_{\omega}=0, \frac{1}{2} N_{b}+\frac{1}{2} N_{v}=1$, with exactly one near defect on each component of $\bar{\Gamma}_{H}$, say $c_{1}^{*}, c_{2}^{*}$, and $E_{\varepsilon_{n}}\left(p_{\varepsilon_{n}} ; \Omega_{s}\right) \leq$ $\pi|\ln s|+C_{2}$. Assume that $c_{1}^{*}$ or $c_{2}^{*}$ is in $\mathcal{V}$, then by Lemma 3.4 we would have: $\left.\left.E_{\varepsilon_{n}}\left(p_{\varepsilon_{n}} ; \Omega \backslash\left(B_{s}\left(c_{1}^{*}\right) \cup B_{s}\left(c_{2}^{*}\right)\right)\right)\right) \geq E_{\varepsilon_{n}}\left(p_{\varepsilon_{n}} ; A_{s, s_{0}}\left(c_{1}^{*}\right) \cup A_{s, s_{0}}\left(c_{2}^{*}\right)\right)\right) \geq \frac{3}{2} \pi \ln \frac{s_{0}}{s}+C$, but, for $s$ small, this would be in contradiction with $E_{\varepsilon_{n}}\left(p_{\varepsilon_{n}} ; \Omega_{s}\right) \leq \pi|\ln s|+C_{2}$. We then conclude that the points are on opposite sides of $\Gamma_{H}$.

THEOREM 4.3. Let $p^{\varepsilon}$ be a minimizer of $E_{\varepsilon}$. For any sequence $\varepsilon \rightarrow 0$, there is a subsequence $\varepsilon_{n} \rightarrow 0$, and two points $\left\{b_{1}^{*}, b_{2}^{*}\right\}$ on opposite sides of $\Gamma_{H}$, such that $p^{\varepsilon_{n}} \rightarrow p^{*}$ in $H_{l o c}^{1} \cap C_{l o c}\left(\bar{\Omega} \backslash\left\{b_{1}^{*}, b_{2}^{*}\right\}\right)$, and $p^{*}: \bar{\Omega} \backslash\left\{b_{1}^{*}, b_{2}^{*}\right\} \rightarrow \mathbb{S}^{1}$ satisfies the equation

$$
\Delta p^{*}+\left|\nabla p^{*}\right|^{2} p^{*}=\sigma\left(\sigma_{s} p_{2}^{*} p^{*}-\mathbf{e}\right) .
$$

Furthermore, $p^{*}$ is a piecewise constant vector field on $\bar{\Gamma}_{H} \backslash\left\{b_{1}^{*}, b_{2}^{*}\right\}$, with values $\pm \mathbf{e}_{1}$; and $p^{*}=\nu$ on $\Gamma_{V}$. And, $\sigma_{s} p_{2}^{*} \geq 0$ a.e. for $\sigma \neq 0$. Recall that $\sigma_{s}=\operatorname{sign}\left(E_{e x}\right)$.

Proof. Consider the sequence $\left\{\varepsilon_{n}\right\}$, and the points $b_{1}^{*}, b_{2}^{*}$, which lie on opposite sides of $\Gamma_{H}$, found in Lemma 4.2; and, for $0<s<s_{0}$, let $\Omega_{s}^{*}=\Omega \backslash\left(B_{s}\left(b_{1}^{*}\right) \cup B_{s}\left(b_{2}^{*}\right)\right)$. Since Lemma 4.2 implies $E_{\varepsilon}\left(p_{\varepsilon}, \Omega_{s}^{*}\right) \leq C(s)$, weakly convergence in $H_{l o c}^{1}\left(\bar{\Omega} \backslash\left\{b_{1}^{*}, b_{2}^{*}\right\}\right)$, and in $W^{1, q}$ for $q<2$ is obtained as in [32], while the stronger convergence follows as 
in [5] and [29] (see also [2]). To show (4.3), we take the exterior product of equation (2.4) with $p_{k}$, and pass to the limit as $k \rightarrow \infty$, to gather $-\Delta p^{*} \times p^{*}+\sigma_{s} \sigma p_{1}^{*}=$ $\lim _{k \rightarrow \infty}-\Delta p_{k} \times \nabla p_{k}+\sigma_{s} \sigma\left(p_{k}\right)_{1}=0$. But, from $\left|p^{*}\right|=1$ on $\bar{\Omega} \backslash\left\{b_{1}^{*}, b_{2}^{*}\right\}$, we have $\left|\nabla p^{*}\right|^{2}+p_{1}^{*} \Delta p_{1}^{*}+p_{2}^{*} \Delta p_{2}^{*}=0$, so $\Delta p^{*}+\left|\nabla p^{*}\right|^{2} p^{*}=\sigma\left(\sigma_{s} p_{2}^{*} p^{*}-\mathbf{e}\right)$. Finally, Mazur's lemma [35, page 120] and Lemma 2.3 imply $\sigma_{s} p_{2}^{*} \geq 0$ a.e. for $\sigma \neq 0$.
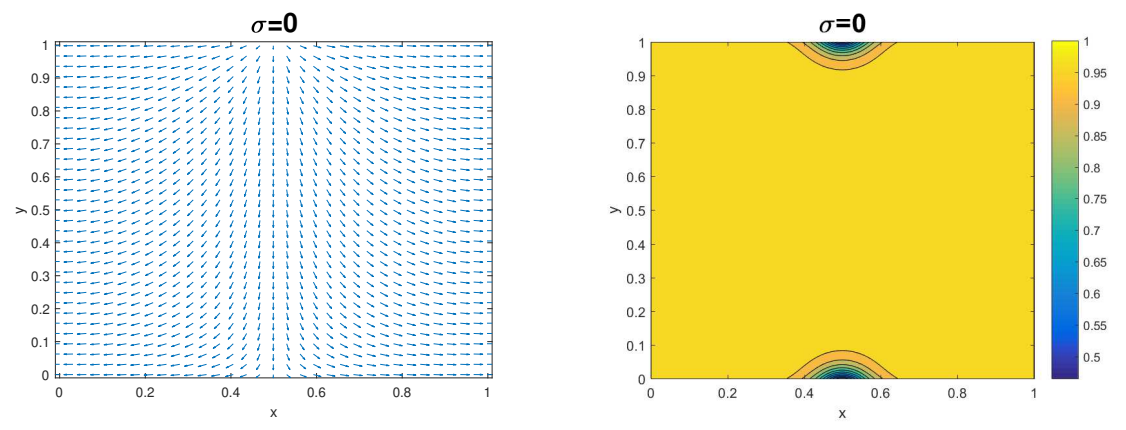

FIG. 3. Numerical solution of the gradient flow (5.1) for $\sigma=0$, with $\varepsilon=0.03$. The left figure depicts the polarization field, the right the contour map of $|p|$.

5. Numerical simulations. We consider the gradient flow (in $L^{2}$ ) of the energy (2.2), and study the behavior of the solutions of the following gradient flow equations:

$$
\begin{aligned}
& \frac{1}{|\ln \varepsilon|} \frac{\partial p}{\partial t}=\Delta p-\frac{|p|^{2}-1}{\varepsilon^{2}} p-\sigma(p-\mathbf{e}), \quad \text { in } \Omega \\
& \frac{\partial p}{\partial \nu}+\frac{1}{\varepsilon}(p \cdot \nu) \nu=0, \quad \text { on } \Gamma_{H} ; \quad p=\nu, \quad \text { on } \Gamma_{V} .
\end{aligned}
$$

We use a random $S^{1}$-valued vector as an initial condition, and a Fourier spectral discretization in the $x$ and $y$ directions, computed using the FFTW libraries [10]. For the time discretization, we use an implicit scheme. Setting $N(p)=\left(1-|p|^{2}\right) p / \varepsilon^{2}$. Given $p^{k}$, we solve: $\frac{1}{\mid \ln \varepsilon} \frac{3 p^{k+1}-4 p^{k}+p^{k-1}}{2 \Delta t}=\Delta p^{k+1}-\sigma p^{k+1}+\sigma \mathbf{e}+2 N\left(p^{k+1}\right)-N\left(p^{k}\right)$.

There has been extensive work done in the dynamics of vortices in a smooth domain, for $\sigma=0$. In the first analytical study of motion of vortices, [4], the authors consider the gradient flow of the classic Ginzburg-Landau energy in $\mathbb{R}^{2}$ (with $\varepsilon=1$ ) for $t \rightarrow \infty$. In [30], the authors prove that the speed of a vortex is $\mathcal{O}(1 /|\ln \varepsilon|)$; they also analyze the creation of a pair of vortices of degree \pm 1 , by constructing specific initial data, which are expected to annihilate each other in finite time. This is what we observe in our simulations, following the formation of two interior vortices, Figure 5.

Figure 3 shows the polarization field plots, and the contour map of $|p|$ at $\sigma=0$. As initial condition we use random unit vectors. As expected from Theorem 4.3, two boundary vortices appear to accommodate the opposite directions of the boundary values on the vertical sides, and the preference for the tangential direction along the horizontal part of the boundary. The polarization points downward in Figure 3, but since up or down states have the same energy cost (Lemma 2.3), the same behavior would be observed in the reverse picture, with polarization now pointing upward.

To understand how the polarization reorients under the effect of the reversed field, we investigate the switching dynamics proceeding as follows: we first obtain the polarization profile of the equilibrium state for $\sigma=70$ (Figures 5 (a) and 6 (a)), then we impose it as the initial value for $\sigma=-70$. Figure 5 demonstrates the switching mechanism and Figure 6 depicts the contour map of $|p|$ showing the vortex dynamics. 


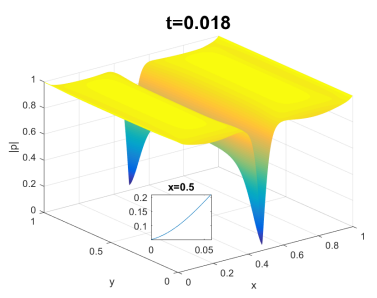

(a)

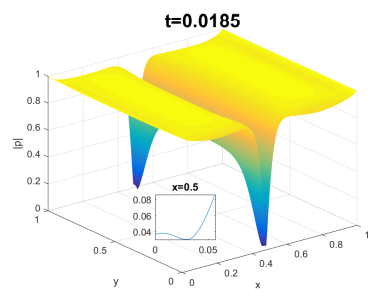

(b)

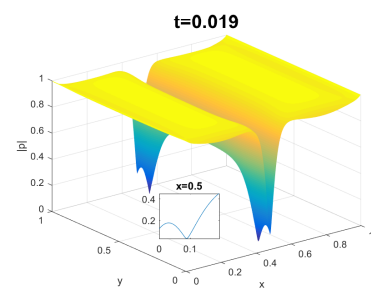

(c)

FIG. 4. Numerical solution of the gradient flow (5.1) for switching dynamics from $\sigma=70$ to $\sigma=-70$, with $\varepsilon=0.03$. The figures show the surface map of $|p|$ at time $t=0.018, t=0.0185$, and $t=0.019$.

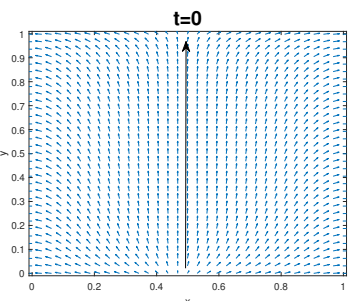

(a)

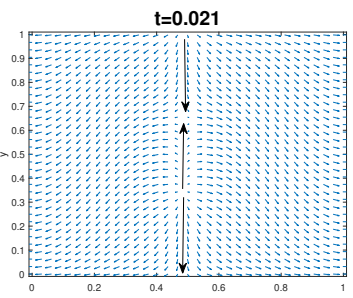

(d)

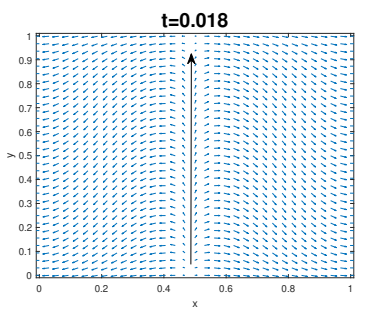

(b)

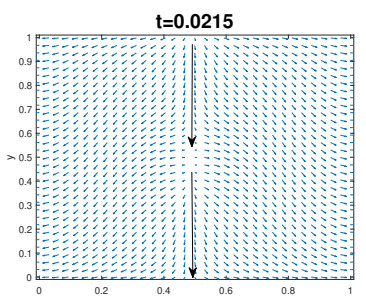

(e)

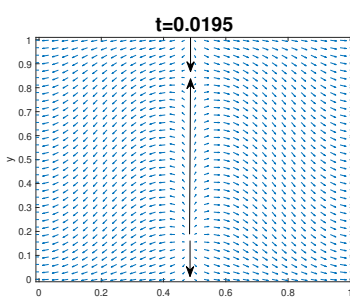

(c)

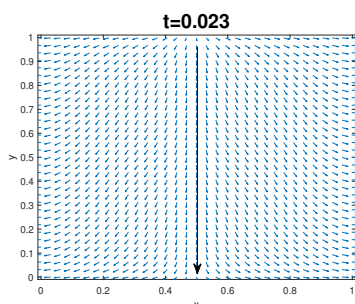

(f)

FIG. 5. Numerical solution of the gradient flow (5.1) for switching dynamics from $\sigma=70$ to $\sigma=-70$, with $\varepsilon=0.03$. The polarization fields are depicted at selected times $t=0, t=$ $0.018, t=0.0195, t=0.021, t=0.0215, t=0.023$.

Figure 4 shows the boundary vortex splitting process that occurs approximately at time $t=0.0185$. The inset figure provided is the plot of $|p|$ near $x=0.5$ and for $0 \leq y \leq 0.05(0 \leq y \leq 0.2$ for (c)). In (a) we show the initial boundary vortices, also depicted in Figures 5 (b) and 6 (b), showing half a +1 boundary vortex near $(0.5,0)$, and half a -1 vortex near $(0.5,1)$. Figure $4(\mathrm{~b})$ shows how the half +1 vortex at $(0.5,0)$ splits into a half -1 vortex at the boundary, and $a+1$ interior vortex. Similarly, the half -1 vortex at $(0.5,1)$ splits into half a +1 boundary vortex, and a -1 interior vortex. This can be seen in Figures 5 (c) and 6 (c) as well. Subsequently, these \pm 1 interior vortices start moving toward the center, see Figure 4(c). Finally, the vortices meet at the center and annihilate each other, as shown in Figure 6 (e).

The electric field strength $\sigma$ needs to be large enough to switch the polarization, since, it needs to support a pair of boundary vortices and the nucleation of two interior vortices. Considering the cost of a boundary vortex, $\frac{\pi}{2}|\ln \varepsilon|$, and of an interior vortex, $\pi|\ln \varepsilon|$, for switching one needs $\sigma / 2 \approx 3 \pi|\ln \varepsilon|$. In fact, with $\varepsilon=0.03$, thus $6 \pi|\ln \varepsilon| \approx 66$, we observe that the switching occurs with $\sigma=70$, but not with $\sigma=60$.

To summarize: once a sufficiently large electric field is reversed, our numerical 


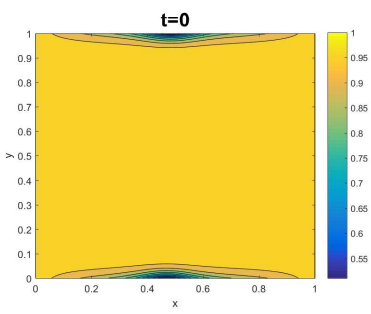

(a)

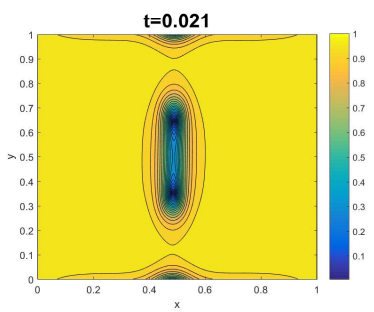

(d)

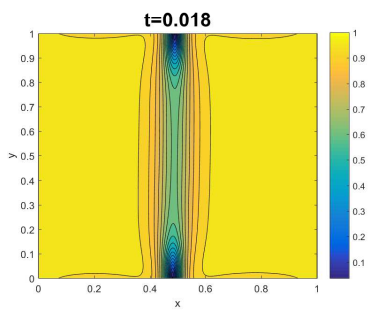

(b)

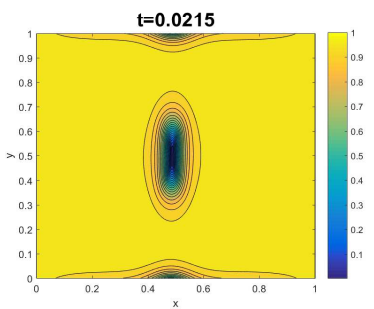

(e)

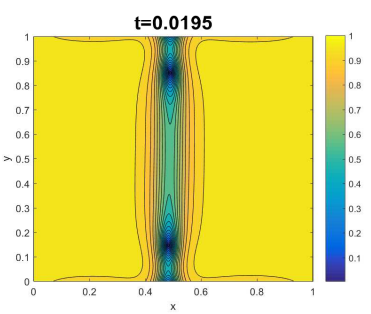

(c)

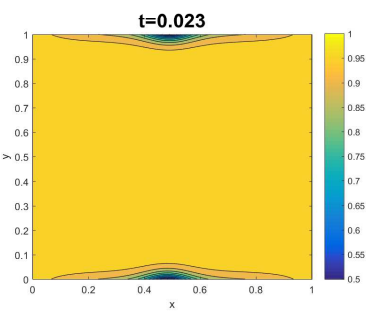

(f)

FIG. 6. Numerical solution of the gradient flow (5.1) for switching dynamics from $\sigma=70$ to $\sigma=-70$, with $\varepsilon=0.03$. The contour map of $|p|$ are shown at the same selected times as in Figure 5.

gradient flow shows the formation of two interior vortices. When the polarizations near the boundary have switched (Figures 5 (c) and 6 (c)), the two interior vortices move along the center line of the square domain (Figure 6(d)(e)), as this allows reversed polarizations in larger regions (Figure $5(\mathrm{~d})(\mathrm{e})$ ). Then, they collide and disappear, leading to the reversed polarizations in the entire domain (Figures 5 (f) and 6 (f)).

6. Acknowledgement. The authors would like to express their thanks to Daniel Phillips, Lia Bronsard, Stan Alama and Dmitry Golovaty for helpful discussions. And, to the anonymous referees for their insightful comments and suggestions.

\section{REFERENCES}

[1] S. Alama, L. Bronsard, and B. Galvão Sousa. Weak anchoring for a two-dimensional liquid crystal. Nonlinear Anal., 119:74-97, 2015.

[2] S. Alama, L. Bronsard, and D. Golovaty. Thin film liquid crystals with oblique anchoring and boojums. https://arxiv.org/pdf/1907.04757.pdf, 2019.

[3] C. Bailey, E. C. Gartland, and A. Jákli. Structure and stability of bent core liquid crystal fibers. Phys. Rev. E, 75:031701, Mar 2007.

[4] P. Bauman, C.-N. Chen, D. Phillips, and P. Sternberg. Vortex annihilation in nonlinear heat flow for Ginzburg-Landau systems. European J. Appl. Math., 6(2):115-126, 1995.

[5] F. Bethuel, H. Brezis, and F. Hélein. Ginzburg-Landau vortices, volume 13 of Progress in Nonlinear Differential Equations and their Applications. Birkhäuser Boston, Inc., Boston, MA, 1994.

[6] H.-Y. Chen, R. Shao, E. Korblova, W. Lee, D. Walba, and N. A. Clark. A bistable liquid-crystal display mode based on electrically driven smectic A layer reorientation. Applied Physics Letters, 91(16), OCT 152007.

[7] H.-Y. Chen and J.-S. Wu. A multistable smectic-a liquid-crystal device with low threshold field. Journal of the Society for Information Display, 18(6):415-420, 2010.

[8] N. A. Clark and S. T. Lagerwall. Submicrosecond bistable electro-optic switching in liquid crystals. Applied Physics Letters, 36(11):899-901, 1980.

[9] A. Eremin and A. Jákli. Polar bent-shape liquid crystals - from molecular bend to layer splay and chirality. Soft Matter, 9:615-637, 2013. 
[10] M. Frigo and S.G. Johnson. The design and implementation of FFTW3. Proceedings of the IEEE, 93(2):216 -231, 2005.

[11] C. J. García-Cervera, T. Giorgi, and S. Joo. Dimensional reduction for the ferroelectric smectic A-type phase of bent-core liquid crystals. Preprint, 2019.

[12] D. J. Gardiner, S. M. Morris, and H. J. Coles. High-efficiency multistable switchable glazing using smectic A liquid crystals. Solar Energy Materials and Solar Cells, 93(3):301-306, 2009.

[13] D. Gilbarg and N. S. Trudinger. Elliptic Partial Differential Equations of Second Order. Springer-Verlag Berlin Heidelberg New York Tokyo, 1983.

[14] K. Gornik, M. Čepič, and N. Vaupotič. Effect of a bias electric field on the structure and dielectric response of the ferroelectric smectic- $a$ liquid crystal in thin planar cells. Phys. Rev. E, 89:012501, Jan 2014.

[15] P. Grisvard. Elliptic problems in nonsmooth domains, volume 24 of Monographs and Studies in Mathematics. Pitman (Advanced Publishing Program), Boston, MA, 1985.

[16] L. Guo, E. Gorecka, D. Pociecha, N. Vaupotič, M. Čepič, R. A. Reddy, K. Gornik, F. Araoka, N. A. Clark, D. M. Walba, K. Ishikawa, and H. Takezoe. Ferroelectric behavior of orthogonal smectic phase made of bent-core molecules. Phys. Rev. E, 84(3):031706, 2011.

[17] A. Jàkli, O. D. Lavrentovich, and J. V. Selinger. Physics of liquid crystals of bent-shaped molecules. Rev. Mod. Phys., to appear.

[18] M. Kurzke. Boundary vortices in thin magnetic films. Calc. Var. Partial Differential Equations, 26(1):1-28, 2006.

[19] J. Lagerwall and F. Giesselmann. Current topics in smectic liquid crystals research. ChemPhysChem, 7(1):20-45, 2006.

[20] G. M. Lieberman. Mixed boundary value problems for elliptic and parabolic equations of second order. J. Math. Anal. Appl., 113(2):422-440, 1986.

[21] C. Luo, A. Majumdar, and R. Erban. Multistability in planar liquid crystal wells. Phys. Rev. E, 85:061702, Jun 2012.

[22] A. Majumdar, C. J. P. Newton, J. M. Robbins, and M. Zyskin. Topology and bistability in liquid crystal devices. Phys. Rev. E, 75(5):051703, 2007.

[23] R. Moser. Ginzburg-Landau vortices for thin ferromagnetic films. AMRX Appl. Math. Res. Express, (1):1-32, 2003.

[24] R. Moser. Boundary vortices for thin ferromagnetic films. Arch. Ration. Mech. Anal., 174(2):267-300, 2004.

[25] T. Niori, T. Sekine, J. Watanabe, T. Furukawa, and H. Takezoe. Distinct ferroelectric smectic liquid crystals consisting of banana shaped achiral molecules. J. Mater. Chem., 6:1231$1233,1996$.

[26] M. A. Osipov and G. Pajak. Molecular theory of proper ferroelectricity in bent-core liquid crystals. Eur. Phys. J. E., 37(79):14079, 2014.

[27] L. A. Parry-Jones, R. B. Meyer, and S. J. Elston. Mechanisms of flexoelectric switching in a zenithally bistable nematic device. J. Appl. Phys., 106(1):014510-014510, 2009.

[28] R. A. Reddy, C. Zhu, R. Shao, E. Korblova, T. Gong, Y. Shen, E. Garcia, M. A. Glaser, J. E. Maclennan, D. M. Walba, and N. A. Clark. Spontaneous ferroelectric order in a bent-core smectic liquid crystal of fluid orthorhombic layers. Science, 332(6025):72-77, 2011.

[29] T. Rivière. Asymptotic analysis for the Ginzburg-Landau equations. Boll. Unione Mat. Ital. Sez. B Artic. Ric. Mat., 8:379-403, 1999.

[30] J. Rubinstein and P. Sternberg. On the slow motion of vortices in the Ginzburg-Landau heat flow. SIAM J. Math. Anal., 26(6):1452-1466, 1995.

[31] E. Sandier. Lower bounds for the energy of unit vector fields and applications. J. Funct. Anal., 152:379-403, 1998.

[32] M. Struwe. On the asymptotic behavior of minimizers of the Ginzburg-Landau model in 2 dimensions. Differential Integral Equations, 7(5-6):1613-1624, 1994.

[33] H. Takezoe and A. Eremin. Bent-Shaped Liquid Crystals: Structures and Physical Properties. CRC Press, Taylor \& Francis Group, 2017.

[34] A. Wagner. Pohozaev's identity from a variational viewpoint. J. Math. Anal. Appl., 266(1):149159, 2002.

[35] K. Yosida. Functional Analysis. Springer-Verlag Berlin Heidelberg New York, sixth edition edition, 1980

[36] C. Zhu, R. Shao, R. A. Reddy, D. Chen, Y. Shen, T. Gong, M. A. Glaser, E. Korblova, P. Rudquist, J. E. Maclennan, D. M. Walba, and N. A. Clark. Topological ferroelectric bistability in a polarization-modulated orthogonal smectic liquid crystal. Journal of the American Chemical Society, 134(23):9681-9687, 2012. PMID: 22545731. 